\title{
ANALYSIS OF THE RESPONSE MATRIX FOR AN EXTENDED TARGET
}

\author{
HONGKAI ZHAO*
}

\begin{abstract}
In this paper we study the response matrix obtained from inter-element response of an active array of transducers that can send out signals and record reflected signals. In particular we analyze the eigenvalues and eigenvectors of the response matrix corresponding to the acoustic field reflected by an extended target, the size of which is comparable to the wavelength. We show that the response matrix has a full rank and the eigenvalues are not well separated even for a single extended target in general. However, when both the size of the target and the size of the active array are small compared to the distance from the array to the target it is shown that the eigenvalues are well separated and that the leading eigenvalues and eigenvectors can be characterized in terms of the location and dimension of the target. Numerical experiments are presented to verify the analysis.
\end{abstract}

Keywords: active array, response matrix, time reversal, Green's function

1. Introduction. Active arrays have been used and studied in many applications such as medical imaging, non-destructive testing, seismic imaging, and target detection and recognition for sonar or radar systems. The type of signals (or wave fields) and devices varies in applications. For example, in medical imaging ultrasound is mostly used, in sonar systems or underwater communications acoustic waves are typically used, in radar system or wireless communications electromagnetic waves are used, and optics or laser may be used in other applications. The typical setup is illustrated in Figure 1.1. The most important function of the active array is that each element in the array can both send out a signal and record the reflected signal. Such an active array can be used to probe a medium by sending out waves to illuminate reflective targets. Information about the targets can be extracted from the reflected signal. One of the key observation, which is explained in next section, is that the reflected signal recorded at the array is related to the output signal of the array by a matrix, the response matrix. In many applications the response matrix can be obtained from the inter-element response, i.e., the response received at one transducer corresponding to an impulse sent out from another transducer. Moreover the product of the response matrix and its adjoint corresponds to the time reversal operator that has been studied extensively in $[14,13,12,11,15,16,3,4,6,1]$. Understanding the structure of the response matrix, such as its eigenvalues and eigenvectors, is crucial for applications using active arrays.

In $[14,13,12]$, an iterative time reversal procedure is proposed and analyzed for the detection of and selective focusing on targets. After recording the reflected signal, reversing it in time, and then sending it out to the medium for a few iterations, the wave field will automatically focus on the "strongest" scatterer. The whole physical procedure can be viewed as a power method for finding the leading eigenvector of the response matrix. For well resolved point scatterers, sizes of which are small compared to the wavelength, it can be shown that each eigenvector of the response matrix corresponds to the wave field at the array due to a point source located at one of the scatterers. Instead of a physical iterated time reversal procedure, which can generate a physical wave field that focuses on a selective target, we can also analyze the response matrix on a computer for the detection or imaging of targets. For example, singular value decomposition of the response matrix and subspace projection were used in [6] for detecting the locations of targets. In all these methods and their analysis the scatterers are considered as point-like

\footnotetext{
*Department of Mathematics, University of California, Irvine, CA 92697-3875; zhao@math.uci.edu. The research is supported by ONR grant N00014-02-1-0090, DARPA grant N00014-02-1-0603 and Sloan Fellowship Foundation.
} 
scatterers so that the response matrix can be cleanly decomposed as the tensor product of the Green's function corresponding to each scatterer.

In this paper we will study the eigenvalues and eigenvectors of the response matrix corresponding to a single extended target. We show that in some asymptotic regimes, the leading eigenvalues and eigenvectors are well separated into groups and can be characterized in terms of the location and dimension of the target. Numerical tests match well with our analysis and show that our formulas can work well in more general situations. It should be pointed out that in many applications the eigenvalues and eigenvectors of the response matrix may also depend on material properties and wave fields, e.g. [3, 4]. In the future we will study multiple targets, different design of the arrays, imaging procedures for both locations and sizes of extended targets, and the effect of random inhomogeneity in the medium and self-averaging in time domain.

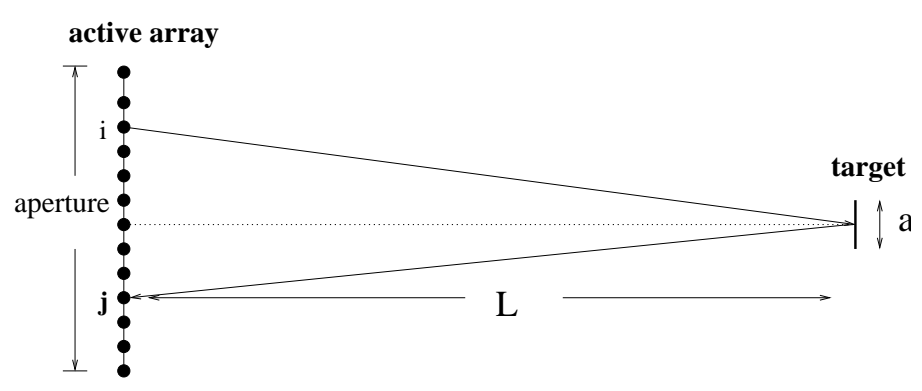

FIG. 1.1. the setup of an active array

Here is the outline of this paper. First, the response matrix and its basic properties for point scatterers are briefly reviewed in section 2 . In section 3 the eigenvalues and eigenvectors of the response matrix corresponding to an extended scatter are analyzed in some asymptotic regimes. In section 4 we study the effect of alignment between the active array and the target. Finally we show numerical experiments to verify our analysis in section 5 .

2. The response matrix of an active array. Define the inter-element response $p_{i j}(t)$ to be the reflected signal at $j-t h$ transducer corresponding to an impulse sent out from $i-t h$ transducer. For an array consisting of $N$ transducers, the matrix $P(t)=$ $\left[p_{i j}(t)\right]_{N \times N}$ is called the response matrix. If the medium is static we have $p_{i j}(t)=p_{j i}(t)$ due to spatial reciprocity. If we assume the medium and the array response is linear, for an output signal $\vec{e}(t)=\left[e_{1}(t), e_{2}(t), \ldots, e_{N}(t)\right]^{T}$, where $e_{i}(t)$ is the output signal at $i-t h$ transducer and $T$ means transpose, the reflected signal at the array,

$$
\vec{r}(t)=\left[r_{1}(t), r_{2}(t), \ldots, r_{N}(t)\right]^{T}=P(t) * \vec{e}(t) .
$$

Here $*$ denotes the convolution in time. The convolution in time domain becomes multiplication in frequency domain,

$$
\vec{r}(\omega)=P(\omega) \vec{e}(\omega)
$$

where $\omega$ is the frequency and $P(\omega)$ is the Fourier transform of $P(t)$. We follow the derivations in $[13,6]$ to illustrate the basic structure of the response matrix $P(\omega)$ for point scatterers in the medium. Denote $G(\boldsymbol{\xi}, \boldsymbol{x})$ to be the Green's function of the medium for frequency $\omega$, which represents the wave field at $\boldsymbol{x}$ for a point source located at $\boldsymbol{\xi}$. Due 
to the spatial reciprocity, $G(\boldsymbol{x}, \boldsymbol{\xi})=G(\boldsymbol{\xi}, \boldsymbol{x})$. Here we suppress the dependence of the Green's function on the frequency.

Assume there are $M$ point scatterers located at $\boldsymbol{x}_{1}, \boldsymbol{x}_{2}, \ldots, \boldsymbol{x}_{M}$ in the medium with reflectivity $\tau_{1}, \tau_{2}, \ldots, \tau_{M}$, if we neglect the multiple scattering among the scatterers, then for a signal $\vec{e}(\omega)=\left[e_{1}(\omega), e_{2}(\omega), \ldots, e_{N}(\omega)\right]^{T}$ sent out from the active array, the reflected signal at $j-t h$ transducer is

$$
r_{j}(\omega)=\sum_{k=1}^{M} \sum_{i=1}^{N} G\left(\boldsymbol{\xi}_{j}, \boldsymbol{x}_{k}\right) \tau_{k} G\left(\boldsymbol{\xi}_{i}, \boldsymbol{x}_{k}\right) e_{i}(\omega),
$$

where $\boldsymbol{\xi}_{1}, \boldsymbol{\xi}_{2}, \ldots, \boldsymbol{\xi}_{N}$ are the locations of the transducers. If we define the illumination vectors, $\vec{g}_{k}, k=1,2, \ldots, M$, to be

$$
\vec{g}_{k}=\left[G\left(\boldsymbol{\xi}_{1}, \boldsymbol{x}_{k}\right), G\left(\boldsymbol{\xi}_{2}, \boldsymbol{x}_{k}\right), \ldots, G\left(\boldsymbol{\xi}_{N}, \boldsymbol{x}_{k}\right)\right]^{T},
$$

i.e., the wave field at the array of transducers corresponding to a point source at the $k-t h$ scatterer, we have

$$
P(\omega)=\sum_{k=1}^{M} \tau_{k} \vec{g}_{k} \vec{g}_{k}^{T} \quad \text { and } \quad \vec{r}(\omega)=P(\omega) \vec{e}(\omega) .
$$

Due to the spatial reciprocity, $P(\omega)$ is symmetric. If we do time reversal, which is phase conjugation in frequency domain, for the reflected signal and send it back to the medium, the new reflected signal is $P(\omega) \overline{P(\omega) \vec{e}(\omega)}$, where ${ }^{-}$denotes complex conjugation. Another phase conjugation gives the second time reversed output signal $\overline{P(\omega)} P(\omega) \vec{e}(\omega)$ in terms of the original output signal $\vec{e}(\omega)$. So $R(\omega)=\overline{P(\omega)} P(\omega)=P^{*}(\omega) P(\omega)$ is called the time reversal matrix (operator), where $*$ denotes the adjoint. $R(\omega)$ is a Hermitian matrix and from (2.1) we have

$$
R(\omega)=\sum_{k=1}^{M} \overline{\tau_{k} \vec{g}_{k} \vec{g}_{k}^{T}} \sum_{k^{\prime}=1}^{M} \tau_{k^{\prime}} \vec{g}_{k^{\prime}} \vec{g}_{k^{\prime}}^{T}=\sum_{k^{\prime}=1}^{M} \sum_{k=1}^{M} \Lambda_{k, k^{\prime}} \overline{\vec{g}}_{k} \vec{g}_{k^{\prime}}^{T},
$$

where

$$
\Lambda_{k, k^{\prime}}=\bar{\tau}_{k} \tau_{k^{\prime}}<\vec{g}_{k}, \vec{g}_{k^{\prime}}>=\bar{\tau}_{k} \tau_{k^{\prime}} \overline{\bar{g}}_{k}^{T} \vec{g}_{k^{\prime}} .
$$

All medium properties are embedded in the Green function in the above formulations. From representations (2.1) and (2.2), we can easily see that both the response matrix $P(\omega)$ and the time reversal matrix $R(\omega)$ are of rank $M$, if $M<N$, and that any eigenvector corresponding to a non-zero eigenvalue is a linear combination of the illumination vectors $\vec{g}_{k}, k=1,2, \ldots, M$. Define the point spread function

$$
\Gamma\left(\boldsymbol{x}^{\prime}, \boldsymbol{x}\right)=\sum_{i=1}^{N} \overline{G\left(\boldsymbol{\xi}_{i}, \boldsymbol{x}^{\prime}\right)} G\left(\boldsymbol{\xi}_{i}, \boldsymbol{x}\right) .
$$

$\Gamma\left(\boldsymbol{x}^{\prime}, \boldsymbol{x}\right)$ is exactly the wave field at point $\boldsymbol{x}$ after phase conjugation of the signal received at the active array corresponding to a point source at point $\boldsymbol{x}^{\prime}$ and sending it back to the medium. The scatterers are well resolved by the active array means

$$
\Gamma\left(\boldsymbol{x}_{k}, \boldsymbol{x}_{k^{\prime}}\right)=\overline{\vec{g}_{k}^{T}} \vec{g}_{k^{\prime}} \approx 0 \text { if } k \neq k^{\prime}
$$


i.e., the wave field corresponding to the time reversal of a point source at one scatterer is almost zero at all other scatterers. Then $\vec{g}_{k}\left(\overline{\vec{g}_{k}}\right)$ is the left (right) singular vectors for $P(\omega)$ with singular values $\tau_{k}\left\|\vec{g}_{k}\right\|^{2}$ since

$$
P(w) \overline{\vec{g}_{k}}=\tau_{k}\left\|\vec{g}_{k}\right\|^{2} \vec{g}_{k}, \quad P^{*}(w) \vec{g}_{k}=\bar{\tau}_{k}\left\|\vec{g}_{k}\right\|^{2} \overline{\vec{g}}_{k} .
$$

Similarly it can be shown that $\overline{\vec{g}_{k}}$ is the eigenvector for the Hermitian matrix $R(\omega)$ with singular value $\left|\tau_{k}\right|^{2}\left\|\vec{g}_{k}\right\|^{4}$. In a homogeneous medium, the focusing property of the point spread function $\Gamma\left(\boldsymbol{x}^{\prime}, \boldsymbol{x}\right)$ is dictated by the diffraction limit, which is proportional to wavelength and propagation distance and is inverse proportional to the size (aperture) of the active array. However if the medium is inhomogeneous and random the resolution of time reversal can beat the diffraction limit. The super-resolution phenomenon is both observed in experiments $[7,5,8,9,10]$ and theoretically analyzed in $[7,2]$. It is shown in [2] that the effective aperture can be much larger than the physical size of the array due to multi-pathing in an inhomogeneous medium and the super-resolution for time reversal is statistically stable in time domain due to self-averaging of different frequencies in a broadband signal.

In $[14,13,12]$, a physical iterative time reversal procedure (D.O.R.T) was used to focus selectively on reflective targets in a real medium. The procedure is equivalent to the power method for finding the leading eigenvector of the time reversal matrix. Since physical time reversal is used, we do not need to know the medium. However, in order for the selective focusing, the targets have to be well resolved by the active array. This procedure is useful for automatic target detection/destruction in practice. In [6] an algorithm for imaging point targets in the medium on computers using an active array was developed. However all these formulations and analysis assume the targets are point scatterers so that the response matrix and time reversal matrix has the simple structure in (2.1) and (2.2) respectively. In this paper we will analyze the eigenvalues and eigenvectors of the response matrix corresponding to an extended target.

3. The singular value decomposition of the response matrix for an extended target. In general the response matrix for an extended target has a full rank and does not have a simple decomposition as in the case for point targets. To simplify the analysis, we assume that each transducer of the active array can be viewed as a point source and the target is a perfect reflector with a normal reflectivity that is equal to 1 . In this case the scattered field can be represented as an integral over the illuminated surface. So the response matrix can be written as

$$
P_{i j}(\omega)=\int_{\Omega} G\left(\boldsymbol{\xi}_{i}, \boldsymbol{x}\right) G\left(\boldsymbol{\xi}_{j}, \boldsymbol{x}\right) \tau\left(\boldsymbol{x} ; \boldsymbol{\xi}_{i}, \boldsymbol{\xi}_{j}\right) d
$$

where $\Omega$ is the part of the surface that can be illuminated by the active array, and $\tau\left(\boldsymbol{x} ; \boldsymbol{\xi}_{i}, \boldsymbol{\xi}_{j}\right)$ is a reflectivity kernel that depends on the incidence and outgoing angle, i.e., the angle between the normal of the surface at $\boldsymbol{x}$ and the vectors $\boldsymbol{\xi}_{i}-\boldsymbol{x}$ and $\boldsymbol{\xi}_{j}-\boldsymbol{x}$ respectively.

In many applications, such as target detections using sonar or radar system, wireless or underwater communications, and geophysics imaging, the distance between the target and the active array is much larger than the wavelength or sizes of the target and array. Let $L$ be the distance between the array and the target, $s$ be the size of the array, $a$ be the size of the target, and $k=\frac{\omega}{c}$ be the wave number, where $c$ is the wave speed. In our study we consider the case where

- the wavelength is comparable to the size of the array and the size of the extended target, i.e., $k a \sim O(1), k s \sim O(1)$; 
- the wavelength is small compared to the distance between the array and the target, i.e., $\frac{1}{k L} \sim \frac{s}{L} \sim \frac{a}{L} \sim o(1)$.

In this case the wave from a transducer is almost planar when it reaches the target. Furthermore we assume the target is a planar target and lies in a plane that is parallel to the plane of the array. Since the size of the array and the size of the target is much smaller than the propagation distance, both the incidence and outgoing angles are small. We first neglect the reflectivity kernel and approximate the response matrix by

$$
P_{i j}(\omega)=\int_{\Omega} G\left(\boldsymbol{\xi}_{i}, \boldsymbol{x}\right) G\left(\boldsymbol{\xi}_{j}, \boldsymbol{x}\right) d \boldsymbol{x}
$$

We will put in a reflectivity kernel later.

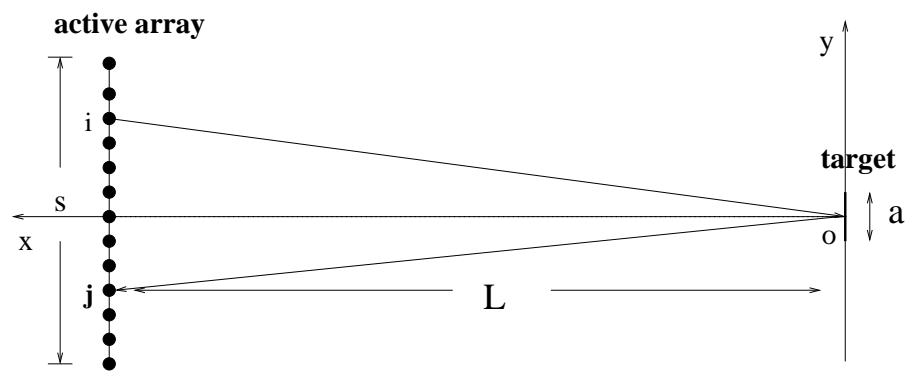

FIG. 3.1

For simplicity we start with a one dimensional target and array in a homogeneous medium as illustrated in Figure 3.1. we expand the Green's function $G\left(\boldsymbol{\xi}_{i}, \boldsymbol{x}\right)$ in free space at a point $\boldsymbol{x}=(0, y)$ on the target in powers of $\frac{1}{L}$. The expansion actually involves powers of $k a, k s$, which are $O(1)$, and powers of $\frac{a}{L}, \frac{s}{L}$, which are $o(1)$.

$$
\begin{aligned}
& G\left(\boldsymbol{\xi}_{i}, \boldsymbol{x}\right)=\frac{e^{i k\left|\boldsymbol{\xi}_{i}-\boldsymbol{x}\right|}}{4 \pi\left|\boldsymbol{\xi}_{i}-\boldsymbol{x}\right|} \frac{e^{i k \sqrt{L^{2}+\left(\eta_{i}-y\right)^{2}}}}{4 \pi \sqrt{L^{2}+\left(\eta_{i}-y\right)^{2}}} \\
& =\tilde{G}\left(\boldsymbol{\xi}_{i}, \boldsymbol{o}\right) \frac{e^{\frac{i k\left(-2 \eta_{i} y+y^{2}\right)}{2 L}+O\left(\frac{1}{L^{3}}\right)}}{1-\frac{2 \eta_{i} y-y^{2}}{2 L^{2}+\eta_{i}^{2}}+O\left(\frac{1}{L^{4}}\right)}=\tilde{G}\left(\boldsymbol{\xi}_{i}, \boldsymbol{o}\right) \frac{e^{\frac{i k\left(-2 \eta_{i} y+y^{2}\right)}{2 L}+O\left(\frac{1}{L^{3}}\right)}}{1-\frac{2 \eta_{i} y-y^{2}}{2 L^{2}}+O\left(\frac{1}{L^{4}}\right)}
\end{aligned}
$$

where $\boldsymbol{o}=(0,0)$ is the center of the target, $\boldsymbol{\xi}_{i}=\left(L, \eta_{i}\right)$ is the location of the $i$-th transducer and $\tilde{G}\left(\boldsymbol{\xi}_{i}, \boldsymbol{o}\right)=\frac{e^{i k L\left(1+\frac{\eta_{i}^{2}}{2 L^{2}}\right)}}{4 \pi L\left(1+\frac{\eta_{i}^{2}}{2 L^{2}}\right)}$, is the parabolic approximation of the Green's function $G\left(\boldsymbol{\xi}_{i}, \boldsymbol{o}\right)$. Here we can use the two dimensional Green's function, zero-order Hankel function of the first kind. Instead we use the three dimensional Green's function for simplicity and consistency with later analysis. Further expanding in $\frac{1}{L}$, we have

$$
G\left(\boldsymbol{\xi}_{i}, \boldsymbol{x}\right)=\tilde{G}\left(\boldsymbol{\xi}_{i}, \boldsymbol{o}\right)\left[1+\frac{i k\left(y^{2}-2 \eta_{i} y\right)}{2 L}-\frac{k^{2}\left(y^{2}-2 \eta_{i} y\right)^{2}}{8 L^{2}}-\frac{y^{2}-2 \eta_{i} y}{2 L^{2}}+O\left(\frac{1}{L^{3}}\right)\right]
$$


Now the response matrix becomes

$$
\begin{aligned}
& P_{i j}(k)=\int_{-\frac{a}{2}}^{\frac{a}{2}} G\left(\boldsymbol{\xi}_{i}, \boldsymbol{x}\right) G\left(\boldsymbol{\xi}_{j}, \boldsymbol{x}\right) d y \\
= & \tilde{G}\left(\boldsymbol{\xi}_{i}, \boldsymbol{o}\right) \tilde{G}\left(\boldsymbol{\xi}_{j}, \boldsymbol{o}\right) \int_{-\frac{a}{2}}^{\frac{a}{2}}\left[1+\frac{i k y^{2}}{L}-\frac{k^{2}\left(\eta_{i}+\eta_{j}-y\right)^{2} y^{2}}{2 L^{2}}+\frac{\left(\eta_{i}+\eta_{j}-y\right) y}{L^{2}}+O\left(\frac{1}{L^{3}}\right)\right] d y \\
(3.5)= & \tilde{G}\left(\boldsymbol{\xi}_{i}, \boldsymbol{o}\right) \tilde{G}\left(\boldsymbol{\xi}_{j}, \boldsymbol{o}\right) \int_{-\frac{a}{2}}^{\frac{a}{2}}\left(1+\frac{i k y^{2}}{L}-\frac{k^{2} y^{4}}{2 L^{2}}-\frac{y^{2}}{L^{2}}\right) d y \\
& -\frac{k^{2}}{L^{2}} \tilde{G}\left(\boldsymbol{\xi}_{i}, \boldsymbol{o}\right) \tilde{G}\left(\boldsymbol{\xi}_{j}, \boldsymbol{o}\right) \eta_{i} \eta_{j} \int_{-\frac{a}{2}}^{\frac{a}{2}} y^{2} d y-\frac{k^{2}}{2 L^{2}} \tilde{G}\left(\boldsymbol{\xi}_{i}, \boldsymbol{o}\right) \tilde{G}\left(\boldsymbol{\xi}_{j}, \boldsymbol{o}\right)\left(\eta_{i}^{2}+\eta_{j}^{2}\right) \int_{-\frac{a}{2}}^{\frac{a}{2}} y^{2} d y \\
& +O\left(\frac{1}{L^{3}}\right)
\end{aligned}
$$

Denote $\alpha(a, k, L)=\int_{-\frac{a}{2}}^{\frac{a}{2}}\left(1+\frac{i k y^{2}}{L}-\frac{k^{2} y^{4}}{2 L^{2}}-\frac{y^{2}}{L^{2}}\right) d y \approx a$. So the response matrix can be decomposed as

$$
P(k)=\alpha(a, k, L) \overrightarrow{\tilde{g}}^{T} \tilde{\tilde{g}}^{T}-\frac{k^{2} a^{3}}{12 L^{2}} \overrightarrow{\tilde{g}}_{1} \overrightarrow{\tilde{g}}_{1}^{T}-\frac{k^{2} a^{3}}{24 L^{2}}\left[\overrightarrow{\tilde{g}}_{2} \overrightarrow{\tilde{g}}^{T}+\overrightarrow{\tilde{g}}_{\overrightarrow{\tilde{g}}}^{2}\right]+O\left(\frac{1}{L^{3}}\right)
$$

where

$$
\begin{gathered}
\overrightarrow{\tilde{g}}=\left[\tilde{G}\left(\boldsymbol{\xi}_{1}, \boldsymbol{o}\right), \tilde{G}\left(\boldsymbol{\xi}_{2}, \boldsymbol{o}\right), \ldots, \tilde{G}\left(\boldsymbol{\xi}_{N}, \boldsymbol{o}\right)\right]^{T} \\
\overrightarrow{\tilde{g}}_{1}=\left[\eta_{1} \tilde{G}\left(\boldsymbol{\xi}_{1}, \boldsymbol{o}\right), \eta_{2} \tilde{G}\left(\boldsymbol{\xi}_{2}, \boldsymbol{o}\right), \ldots, \eta_{N} \tilde{G}\left(\boldsymbol{\xi}_{N}, \boldsymbol{o}\right)\right]^{T} \\
\overrightarrow{\tilde{g}}_{2}=\left[\eta_{1}^{2} \tilde{G}\left(\boldsymbol{\xi}_{1}, \boldsymbol{o}\right), \eta_{2}^{2} \tilde{G}\left(\boldsymbol{\xi}_{2}, \boldsymbol{o}\right), \ldots, \eta_{N}^{2} \tilde{G}\left(\boldsymbol{\xi}_{N}, \boldsymbol{o}\right)\right]^{T}
\end{gathered}
$$

The leading term $a \overrightarrow{\tilde{g}} \overrightarrow{\tilde{g}}^{T}$ corresponds to a point scatter at the center of the extended target with a total reflectivity proportional to the size of the target. Moreover, if the center of the array is aligned with the center of the target, $\overrightarrow{\tilde{g}}$ and $\overrightarrow{\tilde{g}}_{2}$ is even in $\eta$ while $\overrightarrow{\tilde{g}}_{1}$ is odd in $\eta$, we have

$$
\overrightarrow{\tilde{g}}_{1}^{T} \overrightarrow{\vec{g}}=\overrightarrow{\tilde{g}}_{1}^{T} \overrightarrow{\tilde{g}}_{2}=0, \quad \text { and } \quad \overrightarrow{\tilde{g}}_{1}^{T} \overline{\overrightarrow{\tilde{g}}}=\overrightarrow{\tilde{g}}_{1}^{T} \overline{\overrightarrow{\tilde{g}}}_{2}=0
$$

From the orthogonality condition we can separate the second term from the other terms in the matrix decomposition formula (3.6). Moreover since the size of array $s$ is small compared to the distance $L$, the parabolic factor $\frac{\eta^{2}}{L^{2}}$ is very flat and $\vec{g}_{2}$ is approximately aligned with $\vec{g}$. Most of the term, $\frac{k^{2} a^{3}}{24 L^{2}}\left[\overrightarrow{\tilde{g}}_{2} \overrightarrow{\tilde{g}}^{T}+\overrightarrow{\tilde{g}}_{\vec{g}} T_{2}\right]$, is absorbed in the leading term as a small perturbation. In particular the contribution of this term together with other high order terms in $\alpha(a, k, L)$ tend to make the amplitude of the first eigenvalue smaller. This will be verified by numerical experiments in section 5 .

From the analysis, we see that the response matrix has full rank and,

- the dominant eigenvalue is $\lambda_{1} \approx a \overrightarrow{\tilde{g}}^{T} \overrightarrow{\tilde{g}}$ and the associated eigenvector is the illumination vector $\overrightarrow{\tilde{g}}$,

- the second dominant eigenvalue is $\lambda_{2} \approx-\frac{a^{3} k^{2}}{12 L^{2}} \overrightarrow{\tilde{g}}_{1}^{T} \overrightarrow{\tilde{g}}_{1}$ and the associated eigenvector is $\overrightarrow{\tilde{g}}_{1}$.

Now we extend the above analysis to a two dimensional planar target and array as illustrated in figure 3.2, the calculation is similar but messier. First pick a point $\boldsymbol{o}$ on the target as the origin and choose two orthogonal directions in the plane of the target as $y$ 


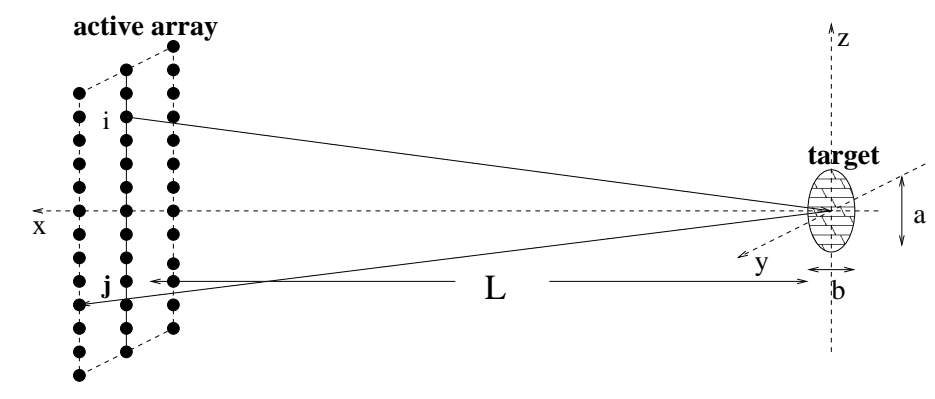

FIG. 3.2.

and $z$ axes. Let $\boldsymbol{x}=(0, y, z)$ denote a point on the target and $\boldsymbol{\xi}_{i}=\left(L, \eta_{i}, \zeta_{i}\right)$ denote the coordinates of the $i$-th transducer. The situation becomes more complicated due to the coupling of different directions. We have

$$
\begin{aligned}
& G\left(\boldsymbol{\xi}_{i}, \boldsymbol{x}\right)=\frac{e^{i k \sqrt{L^{2}+\left(\eta_{i}-y\right)^{2}+\left(\zeta_{i}-z\right)^{2}}}}{4 \pi \sqrt{L^{2}+\left(\eta_{i}-y\right)^{2}+\left(\zeta_{i}-z\right)^{2}}}=\tilde{G}\left(\boldsymbol{\xi}_{i}, \boldsymbol{o}\right) \times \\
& {\left[1+\frac{i k\left(y^{2}-2 \eta_{i} y+z^{2}-2 \zeta_{i} z\right)}{2 L}-\frac{k^{2}\left(y^{2}-2 \eta_{i} y+z^{2}-2 \zeta_{i} z\right)^{2}}{8 L^{2}}-\frac{y^{2}-2 \eta_{i} y+z^{2}-2 \zeta_{i} z}{2 L^{2}}+O\left(\frac{1}{L^{3}}\right)\right] .}
\end{aligned}
$$

where, $\tilde{G}\left(\boldsymbol{\xi}_{i}, \boldsymbol{o}\right)=\frac{e^{i k L\left(1+\frac{\eta_{i}^{2}+\zeta_{i}^{2}}{2 L^{2}}\right)}}{4 \pi L\left(1+\frac{\eta_{i}^{2}+\zeta_{i}^{2}}{2 L^{2}}\right)}$, is again the parabolic approximation of the Green's function, and

$$
\begin{aligned}
& G\left(\boldsymbol{\xi}_{i}, \boldsymbol{x}\right) G\left(\boldsymbol{\xi}_{j}, \boldsymbol{x}\right)=\tilde{G}\left(\boldsymbol{\xi}_{i}, \boldsymbol{o}\right) \tilde{G}\left(\boldsymbol{\xi}_{j}, \boldsymbol{o}\right) \times\left\{1+\frac{i k\left[y^{2}-\left(\eta_{i}+\eta_{j}\right) y+z^{2}-\left(\zeta_{i}+\zeta_{j}\right) z\right)}{L}\right. \\
& \left.-\frac{\left.k^{2}\left[y^{2}-\left(\eta_{i}+\eta_{j}\right) y+z^{2}-\left(\zeta_{i}+\zeta_{j}\right) z\right)\right]^{2}}{2 L^{2}}-\frac{y^{2}-\left(\eta_{i}+\eta_{j}\right) y+z^{2}-\left(\zeta_{i}+\zeta_{j}\right) z}{L^{2}}+O\left(\frac{1}{L^{3}}\right)\right\} .
\end{aligned}
$$


So the response matrix is

$$
\begin{aligned}
& P_{i j}(k)=\int_{\Omega} G\left(\boldsymbol{\xi}_{i}, \boldsymbol{x}\right) G\left(\boldsymbol{\xi}_{j}, \boldsymbol{x}\right) d y d z=\tilde{G}\left(\boldsymbol{\xi}_{i}, \boldsymbol{o}\right) \tilde{G}\left(\boldsymbol{\xi}_{j}, \boldsymbol{o}\right) \times \\
& {\left[\int_{\Omega} 1+\frac{i k\left(y^{2}+z^{2}\right)}{L}-\frac{k^{2}\left(y^{2}+z^{2}\right)^{2}}{2 L^{2}}-\frac{y^{2}+z^{2}}{L^{2}} d y d z\right.} \\
& -\eta_{i} \eta_{j} \int_{\Omega} \frac{k^{2} y^{2}}{L^{2}} d y d z-\zeta_{i} \zeta_{j} \int_{\Omega} \frac{k^{2} z^{2}}{L^{2}} d y d z \\
& -\left(\eta_{i}^{2}+\eta_{j}^{2}\right) \int_{\Omega} \frac{k^{2} y^{2}}{2 L^{2}} d y d z-\left(\zeta_{i}^{2}+\zeta_{j}^{2}\right) \int_{\Omega} \frac{k^{2} z^{2}}{2 L^{2}} d y d z \\
& +\left(\eta_{i}+\eta_{j}\right) \int_{\Omega}-\frac{i k y}{L}+\frac{k^{2}\left(y^{2}+z^{2}\right) y}{L^{2}}+\frac{y}{L^{2}} d y d z \\
& +\left(\zeta_{i}+\zeta_{j}\right) \int_{\Omega}-\frac{i k z}{L}+\frac{k^{2}\left(y^{2}+z^{2}\right) z}{L^{2}}+\frac{z}{L^{2}} d y d z \\
& \left.-\left(\eta_{i} \zeta_{i}+\eta_{i} \zeta_{j}+\eta_{j} \zeta_{i}+\eta_{j} \zeta_{j}\right) \int_{\Omega} \frac{k^{2} y z}{L^{2}} d y d z+O\left(\frac{1}{L^{3}}\right)\right]
\end{aligned}
$$

Denote

$$
\begin{gathered}
\overrightarrow{\tilde{g}}=\left[\tilde{G}\left(\boldsymbol{\xi}_{1}, \boldsymbol{o}\right), \tilde{G}\left(\boldsymbol{\xi}_{2}, \boldsymbol{o}\right), \ldots, \tilde{G}\left(\boldsymbol{\xi}_{N}, \boldsymbol{o}\right)\right]^{T} \\
\overrightarrow{\tilde{g}}_{1 y}=\left[\eta_{1} \tilde{G}\left(\boldsymbol{\xi}_{1}, \boldsymbol{o}\right), \eta_{2} \tilde{G}\left(\boldsymbol{\xi}_{2}, \boldsymbol{o}\right), \ldots, \eta_{N} \tilde{G}\left(\boldsymbol{\xi}_{N}, \boldsymbol{o}\right)\right]^{T} \\
\overrightarrow{\tilde{g}}_{1 z}=\left[\zeta_{1} \tilde{G}\left(\boldsymbol{\xi}_{1}, \boldsymbol{o}\right), \zeta_{2} \tilde{G}\left(\boldsymbol{\xi}_{2}, \boldsymbol{o}\right), \ldots, \zeta_{N} \tilde{G}\left(\boldsymbol{\xi}_{N}, \boldsymbol{o}\right)\right]^{T} \\
\overrightarrow{\vec{g}}_{2 y}=\left[\eta_{1}^{2} \tilde{G}\left(\boldsymbol{\xi}_{1}, \boldsymbol{o}\right), \eta_{2}^{2} \tilde{G}\left(\boldsymbol{\xi}_{2}, \boldsymbol{o}\right), \ldots, \eta_{N}^{2} \tilde{G}\left(\boldsymbol{\xi}_{N}, \boldsymbol{o}\right)\right]^{T} \\
\overrightarrow{\tilde{g}}_{2 z}=\left[\zeta_{1}^{2} \tilde{G}\left(\boldsymbol{\xi}_{1}, \boldsymbol{o}\right), \zeta_{2}^{2} \tilde{G}\left(\boldsymbol{\xi}_{2}, \boldsymbol{o}\right), \ldots, \zeta_{N}^{2} \tilde{G}\left(\boldsymbol{\xi}_{N}, \boldsymbol{o}\right)\right]^{T} \\
\overrightarrow{\tilde{g}}_{2 y z}=\left[\eta_{1} \zeta_{1} \tilde{G}\left(\boldsymbol{\xi}_{1}, \boldsymbol{o}\right), \eta_{2} \zeta_{2} \tilde{G}\left(\boldsymbol{\xi}_{2}, \boldsymbol{o}\right), \ldots, \eta_{N} \zeta_{N} \tilde{G}\left(\boldsymbol{\xi}_{N}, \boldsymbol{o}\right)\right]^{T} .
\end{gathered}
$$

The response matrix can be decomposed as

$$
\begin{aligned}
& P(k)=\overrightarrow{\tilde{g}}^{T} \int_{\Omega} 1+\frac{i k\left(y^{2}+z^{2}\right)}{L}-\frac{k^{2}\left(y^{2}+z^{2}\right)^{2}}{2 L^{2}}-\frac{y^{2}+z^{2}}{L^{2}} d y d z \\
& -\overrightarrow{\tilde{g}}_{1 y} \overrightarrow{\tilde{g}}_{1 y}^{T} \int_{\Omega} \frac{k^{2} y^{2}}{L^{2}} d y d z-\overrightarrow{\tilde{g}}_{1 z} \overrightarrow{\tilde{g}}_{1 z}^{T} \int_{\Omega} \frac{k^{2} z^{2}}{L^{2}} d y d z \\
& -\left(\overrightarrow{\tilde{g}}_{2 y} \overrightarrow{\tilde{g}}^{T}+\overrightarrow{\tilde{g}}_{\tilde{g}_{2 y}}^{T}\right) \int_{\Omega} \frac{k^{2} y^{2}}{2 L^{2}} d y d z-\left(\overrightarrow{\tilde{g}}_{2 z} \overrightarrow{\tilde{g}}^{T}+\overrightarrow{\tilde{g}} \overrightarrow{\tilde{g}}_{2 z}^{T}\right) \int_{\Omega} \frac{k^{2} z^{2}}{2 L^{2}} d y d z \\
& +\left(\overrightarrow{\tilde{g}}_{1 y} \overrightarrow{\tilde{g}}^{T}+\overrightarrow{\tilde{g}}_{\vec{g}_{1 y}^{T}}^{T}\right) \int_{\Omega}-\frac{i k y}{L}+\frac{k^{2}\left(y^{2}+z^{2}\right) y}{L^{2}}+\frac{y}{L^{2}} d y d z \\
& +\left(\overrightarrow{\tilde{g}}_{1 z} \overrightarrow{\tilde{g}}^{T}+\overrightarrow{\tilde{g}}_{\tilde{g}_{1 z}^{T}}^{T}\right) \int_{\Omega}-\frac{i k z}{L}+\frac{k^{2}\left(y^{2}+z^{2}\right) z}{L^{2}}+\frac{z}{L^{2}} d y d z \\
& -\left(\overrightarrow{\tilde{g}}_{1 y} \overrightarrow{\tilde{g}}_{1 z}^{T}+\overrightarrow{\tilde{g}}_{1 z} \overrightarrow{\tilde{g}}_{1 y}^{T}+\overrightarrow{\tilde{g}}_{2 y z} \overrightarrow{\tilde{g}}^{T}+\overrightarrow{\tilde{g}}_{\tilde{g}_{2 y z}^{T}}^{T}\right) \int_{\Omega} \frac{k^{2} y z}{L^{2}} d y d z+O\left(\frac{1}{L^{3}}\right)
\end{aligned}
$$


The above formula shows the decomposition of the response matrix in any coordinate system. Again the leading term corresponds to a point scatter. The decomposition of the response matrix is not obvious from this general expression since the two directions and the center are coupled together. However, if we choose $\boldsymbol{o}$ to be the mass center of the target, we have

$$
\int_{\Omega} y d y d z, \quad \int_{\Omega} z d y d z=0
$$

Now we have an extra freedom of rotation of $y$ and $z$ axes around $\boldsymbol{o}$. Denote the integral $R(\theta)=\int_{\Omega} y z d y d z$ as a function of rotation angle $\theta$. Since $R(\theta)=-R(\theta \pm \pi / 2)$, so we must have at least two $\theta$ such that $\int_{\Omega} y z d y d z=0$. Due to this symmetry and cancellations, the following terms are usually small,

$$
\int_{\Omega} y^{p} z^{q} d y d z \approx 0, \text { for }(p, q) \in\{(1,2),(2,1),(0,3),(3,0)\} .
$$

Hence the decomposition of the response matrix can be simplified as

$$
\begin{aligned}
& P(k) \approx \overrightarrow{\tilde{g}}_{\vec{g}}^{T} \int_{\Omega} d y d z-\frac{k^{2}}{L^{2}} \overrightarrow{\tilde{g}}_{1 y} \overrightarrow{\tilde{g}}_{1 y}^{T} \int_{\Omega} y^{2} d y d z-\frac{k^{2}}{L^{2}} \overrightarrow{\tilde{g}}_{1 z} \overrightarrow{\tilde{g}}_{1 z}^{T} \int_{\Omega} z^{2} d y d z \\
& -\frac{k^{2}}{2 L^{2}}\left[\overrightarrow{\tilde{g}}_{2 y} \overrightarrow{\tilde{g}}^{T}+\overrightarrow{\tilde{g}}_{\tilde{g}^{T}}^{T}\right] \int_{\Omega} y^{2} d y d z-\frac{k^{2}}{2 L^{2}}\left[\overrightarrow{\tilde{g}}_{2 z} \overrightarrow{\tilde{g}}^{T}+\overrightarrow{\tilde{g}}_{2}^{T}{ }_{2 z}^{T}\right] \int_{\Omega} z^{2} d y d z+O\left(\frac{1}{L^{3}}\right) .
\end{aligned}
$$

Again, if the array of transducers is symmetric with respect to the $y$ and $z$ axes, we have the following orthogonality properties defined as before,

$$
\overrightarrow{\tilde{g}}_{1 y} \perp \overrightarrow{\tilde{g}}, \overrightarrow{\tilde{g}}_{2 y}, \quad \overrightarrow{\tilde{g}}_{2 z} \quad \overrightarrow{\tilde{g}}_{1 z} \perp \overrightarrow{\tilde{g}}, \overrightarrow{\tilde{g}}_{2 y}, \overrightarrow{\tilde{g}}_{2 z} \quad \overrightarrow{\tilde{g}}_{1 y} \perp \overrightarrow{\tilde{g}}_{1 z} .
$$

In this case we have:

- the dominant eigenvector is the illumination $\overrightarrow{\tilde{g}}$ with eigenvalue $\lambda_{1} \approx \overrightarrow{\tilde{g}}^{T} \overrightarrow{\tilde{g}} \int_{\Omega}$

- the next two dominant eigenvectors are: $\overrightarrow{\tilde{g}}_{1 y}$ with eigenvalue $\lambda_{2} \approx-\frac{k^{2}}{L^{2}} \overrightarrow{\tilde{g}}_{1 y}^{T} \overrightarrow{\tilde{g}}_{1 y} \int_{\Omega} y^{2}$ and $\overrightarrow{\tilde{g}}_{1 z}$ with eigenvalue $\lambda_{3} \approx-\frac{k^{2}}{L^{2}} \overrightarrow{\tilde{g}}_{1 z} \overrightarrow{\tilde{g}}_{1 z}^{T} \int_{\Omega} z^{2}$.

The two symmetric axes of the target are intrinsic and are independent of the artificial $y$ and $z$ axes we choose. The directions of the two symmetric axes with respect to the artificial $y$ and $z$ axes we choose are embedded in the response matrix and can be extracted from the leading eigenvectors as will be shown later from the numerical experiments in section 5. Essentially these formulas suggest that we can find both the location and size of an extended target as well as the symmetric axes and a few moments with respect to these axes using the leading eigenvalues and eigenvectors of the response matrix. In practice ratios of the eigenvalues are more robust and can be used to determine aspect ratios.

For a three dimensional scatterer for which the Born approximation is valid, the integration over the scatterer will be in three dimensions. Using similar analysis for the response matrix we will get a third eigenvalue and eigenvector in the second group of dominant eigenvalues and eigenvectors that correspond to the third dimension. In practice, the depth information is often relative weak due to small glancing aperture and is more difficult to capture from the response matrix. Especially when the distance between the active array and the target is long, we would see an effective planar shape of the target. In [16], careful numerical simulations are done to analyze the eigenvalues and eigenvectors of the response matrix and illustrate very similar behavior to our results here. 
For medium with weak random inhomogeneity, it is shown that for long propagation distance, the wave field looks like the wave propagation in a homogenized medium. For long propagation distance the Green's function is a modification of the homogeneous Green's function. So the behavior of the response matrix and its eigenvalues and eigenvectors should behave similarly. We will analyze these situations in the future study.

Now we take into account the variation of reflectivity due to different incident and outgoing angles. In particular we choose a special reflectivity kernel

$$
\tau\left(\boldsymbol{x} ; \boldsymbol{\xi}_{i}, \boldsymbol{\xi}_{j}\right)=\cos \theta_{i}(\boldsymbol{x}) \cos \theta_{j}(\boldsymbol{x}),
$$

where $\theta_{i}(\boldsymbol{x})$ is the angle between $\boldsymbol{x}-\boldsymbol{\xi}_{i}$ and the normal at $\boldsymbol{x}$. At a point $\boldsymbol{x}$ on a one dimensional target,

$$
\cos \theta_{i}(\boldsymbol{x})=\frac{L}{\sqrt{L^{2}+\left(\eta_{i}-y\right)^{2}}}=\frac{1}{1+\frac{\eta_{i}^{2}}{2 L^{2}}}\left(1-\frac{y^{2}-2 \eta_{i} y}{2 L^{2}}\right)+O\left(\frac{1}{L^{4}}\right)
$$

If we plug this asymptotic expansion back into the response matrix expression and denote

$$
\breve{G}\left(\boldsymbol{\xi}_{i}, \boldsymbol{o}\right)=\tilde{G}\left(\boldsymbol{\xi}_{i}, \boldsymbol{o}\right) \frac{1}{1+\frac{\eta_{i}^{2}}{2 L^{2}}},
$$

we have

$$
\begin{aligned}
& P_{i j}(k)=\breve{G}\left(\boldsymbol{\xi}_{i}, \boldsymbol{o}\right) \breve{G}\left(\boldsymbol{\xi}_{j}, \boldsymbol{o}\right) \int_{-\frac{a}{2}}^{\frac{a}{2}}\left[1+\frac{i k y^{2}}{L}-\frac{k^{2}\left(\eta_{i}+\eta_{j}-y\right)^{2} y^{2}}{2 L^{2}}+\frac{2\left(\eta_{i}+\eta_{j}-y\right) y}{L^{2}}+O\left(\frac{1}{L^{3}}\right)\right] d y \\
& =\breve{G}\left(\boldsymbol{\xi}_{i}, \boldsymbol{o}\right) \breve{G}\left(\boldsymbol{\xi}_{j}, \boldsymbol{o}\right) \int_{-\frac{a}{2}}^{\frac{a}{2}}\left(1+\frac{i k y^{2}}{L}-\frac{k^{2} y^{4}}{2 L^{2}}-\frac{2 y^{2}}{L^{2}}\right) d y \\
& -\frac{k^{2}}{L^{2}} \breve{G}\left(\boldsymbol{\xi}_{i}, \boldsymbol{o}\right) \breve{G}\left(\boldsymbol{\xi}_{j}, \boldsymbol{o}\right) \eta_{i} \eta_{j} \int_{-\frac{a}{2}}^{\frac{a}{2}} y^{2} d y-\frac{k^{2}}{2 L^{2}} \breve{G}\left(\boldsymbol{\xi}_{i}, \boldsymbol{o}\right) \breve{G}\left(\boldsymbol{\xi}_{j}, \boldsymbol{o}\right)\left(\eta_{i}^{2}+\eta_{j}^{2}\right) \int_{-\frac{a}{2}}^{\frac{a}{2}} y^{2} d y \\
& +O\left(\frac{1}{L^{3}}\right)
\end{aligned}
$$

which is almost exactly the same as equation (3.5). Now if we define the new illumination vectors to be

$$
\begin{gathered}
\overrightarrow{\vec{g}}=\left[\breve{G}\left(\boldsymbol{\xi}_{1}, \boldsymbol{o}\right), \breve{G}\left(\boldsymbol{\xi}_{2}, \boldsymbol{o}\right), \ldots, \breve{G}\left(\boldsymbol{\xi}_{N}, \boldsymbol{o}\right)\right]^{T} \\
\overrightarrow{\breve{g}}_{1}=\left[\eta_{1} \breve{G}\left(\boldsymbol{\xi}_{1}, \boldsymbol{o}\right), \eta_{2} \breve{G}\left(\boldsymbol{\xi}_{2}, \boldsymbol{o}\right), \ldots, \eta_{N} \breve{G}\left(\boldsymbol{\xi}_{N}, \boldsymbol{o}\right)\right]^{T} \\
\overrightarrow{\breve{g}}_{2}=\left[\eta_{1}^{2} \breve{G}\left(\boldsymbol{\xi}_{1}, \boldsymbol{o}\right), \eta_{2}^{2} \breve{G}\left(\boldsymbol{\xi}_{2}, \boldsymbol{o}\right), \ldots, \eta_{N}^{2} \breve{G}\left(\boldsymbol{\xi}_{N}, \boldsymbol{o}\right)\right]^{T}
\end{gathered}
$$

and $\alpha(a, k, L)=\int_{-\frac{a}{2}}^{\frac{a}{2}}\left(1+\frac{i k y^{2}}{L}-\frac{k^{2} y^{4}}{2 L^{2}}-\frac{2 y^{2}}{L^{2}}\right) d y$, we have exactly the same decomposition of the response matrix as in equation (3.6). For a more general reflectivity kernel

$$
\tau\left(\boldsymbol{x} ; \boldsymbol{\xi}_{i}, \boldsymbol{\xi}_{j}\right)=f\left(\theta_{i}(\boldsymbol{x}), \theta_{j}(\boldsymbol{x})\right)=\tilde{f}\left(\frac{1}{\sqrt{1+\left(\frac{\eta_{i}-y}{L}\right)^{2}}}, \frac{1}{\sqrt{1+\left(\frac{\eta_{j}-y}{L}\right)^{2}}}\right),
$$

we can use power expansion in $\frac{1}{L}$ to get the explicit formulas. The extension to two dimensional target is exactly the same. 
4. Alignment of the array and the target. In the above analysis, the geometric decomposition of the response matrix for an extended target utilizes the symmetry and alignment of the active array with the target. In most applications, neither the geometry nor the location of the target is known. Hence the alignment of the active array with the target and how it affects the decomposition of the response matrix is an important question in practice. For a general two dimensional target there are two alignments, one is the alignment of the center and the other is the alignment of the lines of symmetry. The center and symmetry of the target is intrinsic while the center and symmetry of the active array can be maneuvered. In fact, if the geometry of the active array is designed properly, such as has a shape of square or disc, the array is symmetric with respect to any orthogonal coordinate system whose origin is at the center of the array. We will see from numerical tests in section 5 the two symmetric directions are automatically embedded in the eigenvectors of the response matrix and can be found out easily. Now the only issue becomes the alignment of the center of the array and the center of the target.

We study the simple case of a one dimensional target illustrated in Figure 4.1. Our previous decomposition of the response matrix (3.6) is not changed. Hence the leading term in the decomposition is still approximately $a \vec{g}^{T} \vec{g}^{T}$, where $a$ is the size of the target and $\vec{g}$ is the corresponding illumination vector. Now we analyze how much the orthogonality property is violated if the shift in the alignment of the center of the array and the center of the target is small compared to the size of the active array. We will also verify this using numerical experiments in section 5 .

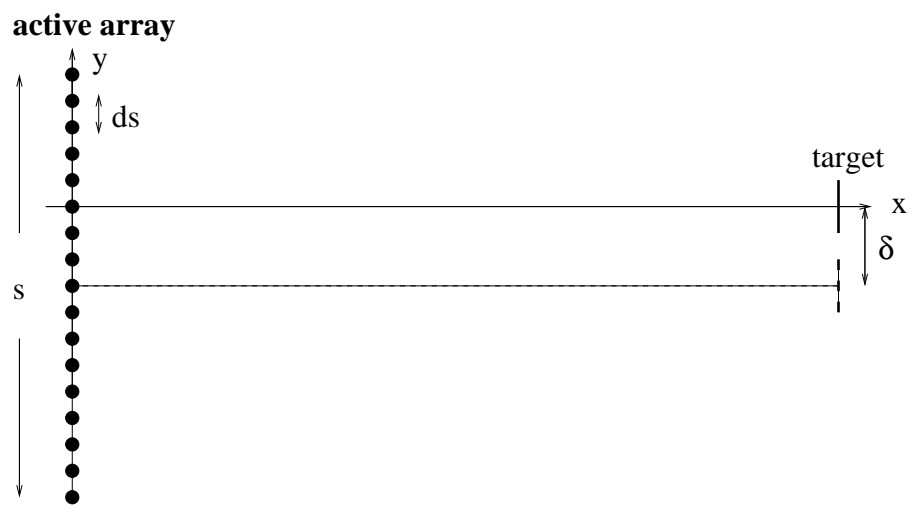

FIG. 4.1

When the distance between the target and the active array $L$ is large compared to the size of the array $s$, i.e., when the aperture $\frac{s}{L}$ is small, we have $G\left(\boldsymbol{\xi}_{i}, \boldsymbol{o}\right) \approx G(\tilde{\boldsymbol{o}}, \boldsymbol{o})$, where $\tilde{\boldsymbol{o}}$ is the center of array and $\boldsymbol{o}$ is the center of the target. So

$$
\begin{gathered}
\overrightarrow{\tilde{g}}=\left[G\left(\boldsymbol{\xi}_{1}, \boldsymbol{o}\right), G\left(\boldsymbol{\xi}_{2}, \boldsymbol{o}\right), \ldots, G\left(\boldsymbol{\xi}_{N}, \boldsymbol{o}\right)\right]^{T} \approx G(\tilde{\boldsymbol{o}}, \boldsymbol{o})[1,1, \ldots, 1]^{T}, \\
\overrightarrow{\tilde{g}}_{1}=\left[\eta_{1} G\left(\boldsymbol{\xi}_{1}, \boldsymbol{o}\right), \eta_{2} G\left(\boldsymbol{\xi}_{2}, \boldsymbol{o}\right), \ldots, \eta_{N} G\left(\boldsymbol{\xi}_{N}, \boldsymbol{o}\right)\right]^{T} \approx G(\tilde{\boldsymbol{o}}, \boldsymbol{o})\left[\eta_{1}, \eta_{2}, \ldots, \eta_{N}\right]^{T}, \\
\overrightarrow{\tilde{g}}_{2}=\left[\eta_{1}^{2} G\left(\boldsymbol{\xi}_{1}, \boldsymbol{o}\right), \eta_{2}^{2} G\left(\boldsymbol{\xi}_{2}, \boldsymbol{o}\right), \ldots, \eta_{N}^{2} G\left(\boldsymbol{\xi}_{N}, \boldsymbol{o}\right)\right]^{T} \approx G(\tilde{\boldsymbol{o}}, \boldsymbol{o})\left[\eta_{1}^{2}, \eta_{2}^{2}, \ldots, \eta_{N}^{2}\right]^{T} .
\end{gathered}
$$

Let $d s$ be the separation space between two adjacent transducers and $|G|=|G(\tilde{\boldsymbol{o}}, \boldsymbol{o})|$, , 
then

$$
\begin{aligned}
& \|\overrightarrow{\tilde{g}}\| \approx \sqrt{\int_{-s / 2-\delta}^{s / 2-\delta}|G|^{2} d y / d s}=|G| \sqrt{\frac{s}{d s}} \\
& \left\|\overrightarrow{\tilde{g}_{1}}\right\| \approx \sqrt{\int_{-s / 2-\delta}^{s / 2-\delta}|G|^{2} y^{2} d y / d s}=|G| \sqrt{\frac{(s / 2-\delta)^{3}+(s / 2+\delta)^{3}}{3 d s}} \\
& \left\|\overrightarrow{\tilde{g}_{2}}\right\| \approx \sqrt{\int_{-s / 2-\delta}^{s / 2-\delta}|G|^{2} y^{4} d y / d s}=|G| \sqrt{\frac{(s / 2-\delta)^{5}+(s / 2+\delta)^{5}}{5 d s}}
\end{aligned}
$$

and

$$
\left|<\frac{\overrightarrow{\tilde{g}}}{\|\overrightarrow{\tilde{g}}\|}, \frac{\overrightarrow{\tilde{g}}_{1}}{\left\|\overrightarrow{\tilde{g}}_{1}\right\|}>\right| \approx \frac{\left.\left|\int_{-s / 2-\delta}^{s / 2-\delta}\right| G\right|^{2} y d y / d s \mid}{\|\overrightarrow{\tilde{g}}\|\left\|\overrightarrow{\tilde{g}}_{1}\right\|}=\frac{\left[(s / 2+\delta)^{2}-(s / 2-\delta)^{2}\right] / 2}{\sqrt{\left[(s / 2-\delta)^{3}+(s / 2+\delta)^{3}\right] s / 3}} \leq 2 \sqrt{3} \frac{\delta}{s},
$$

where

$$
\begin{aligned}
& \alpha^{3}+\beta^{3}=(\alpha+\beta)\left(\alpha^{2}-\alpha \beta+\beta^{2}\right) \\
& \alpha^{2}-\alpha \beta+\beta^{2} \geq(\alpha+\beta)^{2} / 4
\end{aligned}
$$

is used. Similarly we have

$$
\left|<\frac{\overrightarrow{\tilde{g}}}{\|\overrightarrow{\tilde{g}}\|}, \frac{\overrightarrow{\tilde{g}}_{2}}{\left\|\overrightarrow{\tilde{g}}_{2}\right\|}>\right| \approx \frac{\left[(s / 2+\delta)^{4}-(s / 2-\delta)^{4}\right] / 4}{\sqrt{\left[(s / 2-\delta)^{3}+(s / 2+\delta)^{3}\right] / 3} \sqrt{\left[(s / 2-\delta)^{5}+(s / 2+\delta)^{5}\right] / 5}} \leq 2 \sqrt{15} \frac{\delta}{s},
$$

where

$$
\begin{aligned}
& \alpha^{5}+\beta^{5}=(\alpha+\beta)\left(\alpha^{4}-\alpha^{3} \beta+\alpha^{2} \beta^{2}-\alpha \beta^{3}+\beta^{4}\right) \\
& \alpha^{4}-\alpha^{3} \beta+\alpha^{2} \beta^{2}-\alpha \beta^{3}+\beta^{4} \geq\left(\alpha^{2}+\beta^{2}\right)^{2} / 4
\end{aligned}
$$

is used. So the orthogonality condition deteriorates approximately linearly in $\frac{\delta}{s}$. The larger the size of the active array is the more robust the decomposition of the response matrix is. Also it appears from the numerical experiments that the even part of $\overrightarrow{\tilde{g}}_{1}$ is absorbed in the first eigenvector as a perturbation and the odd part of $\overrightarrow{\tilde{g}}_{1}$ becomes the second eigenvector. Since the first term, $a \overrightarrow{\tilde{g}} \overrightarrow{\tilde{g}}^{T}$, in the response matrix decomposition is dominant and more robust to the center shift, we can first use the leading term to estimate the center of the target and then adjust the center of the array towards the estimated center of the target to get better estimation of the size in imaging.

5. Numerical experiments. In this section we use numerical examples to verify our analysis on the leading eigenvalues and eigenvectors of the response matrix for an extended target. In particular we will demonstrate the relation between the eigenvectors and the illumination vectors and verify the formulas for the leading eigenvalues. In our numerical examples, the response matrix of an extended target was formed using the integral

$$
P_{i j}=\int_{\Omega} G\left(\boldsymbol{\xi}_{i}, \boldsymbol{x}\right) G\left(\boldsymbol{\xi}_{j}, \boldsymbol{x}\right) d \boldsymbol{x}
$$

where $\boldsymbol{\xi}_{i}, \boldsymbol{\xi}_{j}$ are the positions of the $i$ th and $j$ th transducers respectively and

$$
G(\boldsymbol{\xi}, \boldsymbol{x})=\frac{e^{i \boldsymbol{k}|\boldsymbol{\xi}-\boldsymbol{x}|}}{4 \pi|\boldsymbol{\xi}-\boldsymbol{x}|}
$$


is the three dimensional Green's function for homogeneous medium. We use a simple quadrature for the integral on the target, denoted by $\Omega$, with a grid size $h$ that resolves both the wavelength and the target. In all our numerical setups we use fixed wavelength $\lambda=0.5 m$ and wavenumber $k=\frac{2 \pi}{\lambda}=4 \pi$. We vary the size of the active array $s$, the propagation distance $L$ and the size of the target relative to the wavelength, i.e., $k a$, to verify our analysis and formulas. The singular value decomposition (SVD) of the response matrix is done by MATLAB. Note that the eigenvectors computed by SVD in MATLAB: (1) are always normalized to have a unit $L_{2}$ norm, and (2) have an arbitrary phase shift. Also the phase plot is up to a $2 \pi$ shift.

In the following, we present numerical examples of one dimensional arrays and targets in 5.1 as well as two dimensional arrays and targets in 5.2.

5.1. One dimensional arrays and targets. In this section we present numerical results in one dimension. We show the spectrum of the response matrix and the asymptotic formulas for the top two eigenvalues as well as the top two eigenvectors and their relations to the illumination vectors.

Example 1: In this example we show the spectrum of the response matrix for a single one dimensional extended target. Figure 5.1 shows loglog plot of the magnitudes of all eigenvalues of the response matrix corresponding to different target size $a$ and propagation distance $L$. The size of the array is $s=10 \mathrm{~m}$ and the transducers are put half wavelength apart, i.e., there are $\frac{2 s}{\lambda}=40$ transducers. We see that the response matrix has a full rank in general and the top two eigenvalues are well separated from each other and from the other eigenvalues in the asymptotic regime which we discussed earlier.
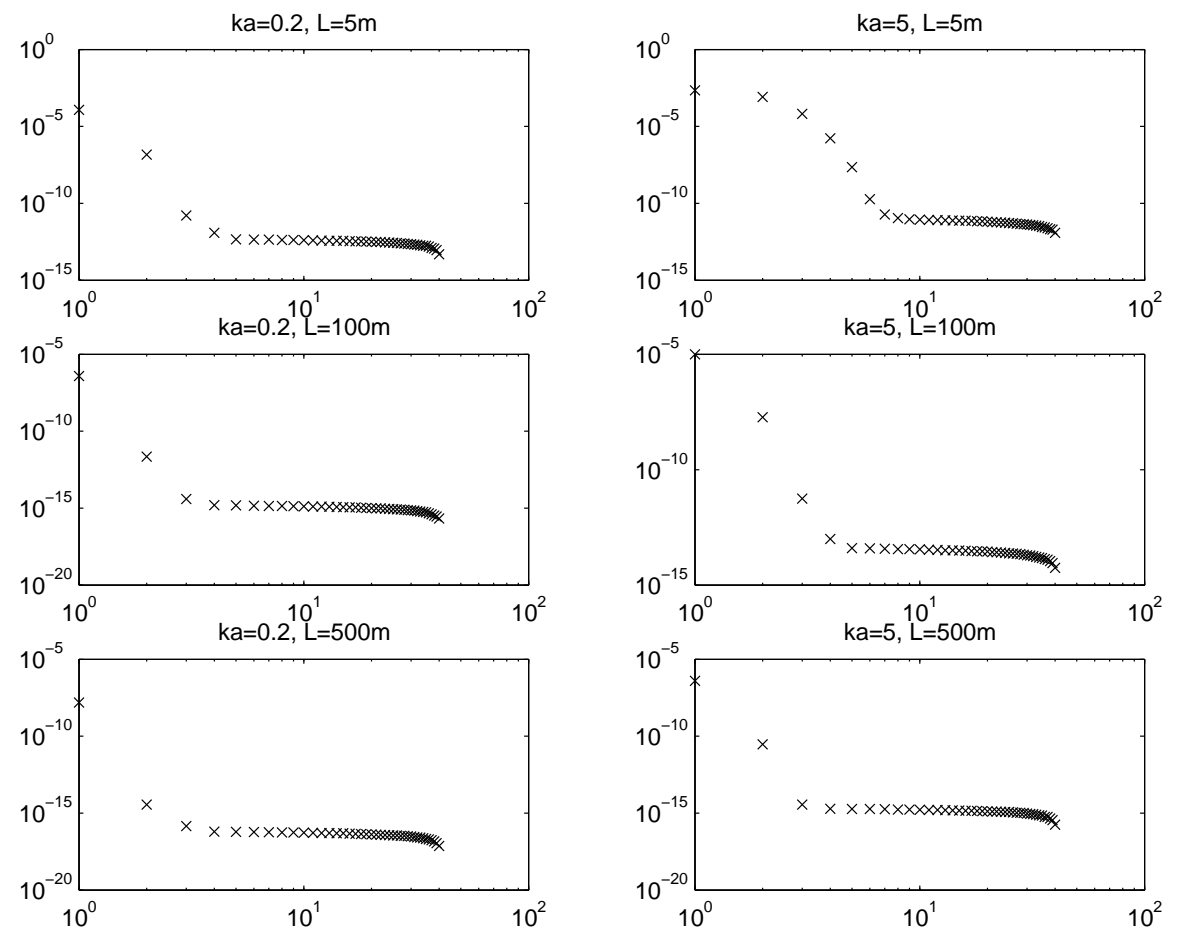

$\mathrm{k}=4 \pi$, aperture $=10 \mathrm{~m}$

FIG. 5.1. Plots of the magnitudes of eigenvalues 
Example 2: In this example we demonstrate the relation between the top two eigenvectors of the response matrix and the corresponding illumination vectors. We also verify the formulas for the top two eigenvalues numerically. The basic setup is illustrated in Figure 3.1 with $L=500 m, k=4 \pi, k a=16, s=40 \mathrm{~m}$. The transducers are half wavelength apart. In Figure 5.2, we plot the magnitude and phase for each component of the top two eigenvectors and compare them to the illumination vectors. Figure 5.2(a) plot the magnitude and phase of the first eigenvector against the illumination vector $\overrightarrow{\tilde{g}}$ defined in (3.7). Figure 5.2(b) plot the magnitude and phase of the second eigenvector against the illumination vector $\overrightarrow{\tilde{g}}_{1}$ defined in (3.7). The correspondence and pattern similarity is striking.
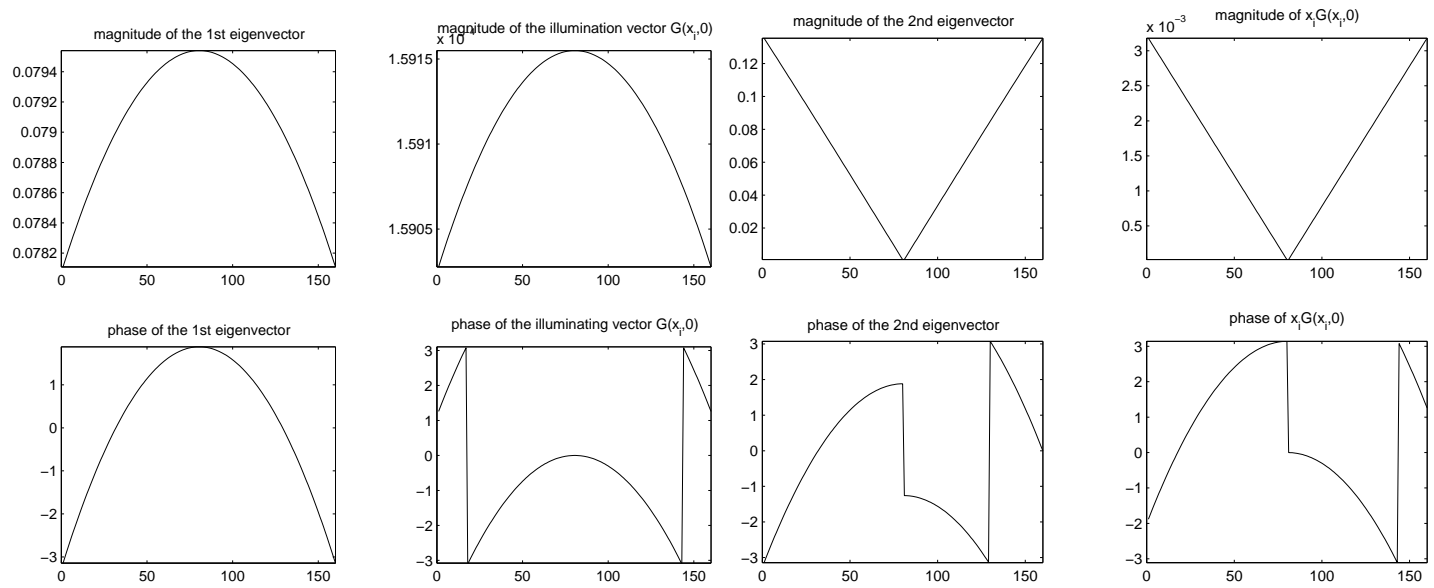

(a) the phase and magnitude of the first eigenvector

(b) the phase and magnitude of the second eigenvector

FIG. 5.2 .

Denote $\vec{v}_{1}, \vec{v}_{2}$ to be the top two eigenvectors with eigenvalues $\lambda_{1}, \lambda_{2}$ computed by the MATLAB, we have numerically:

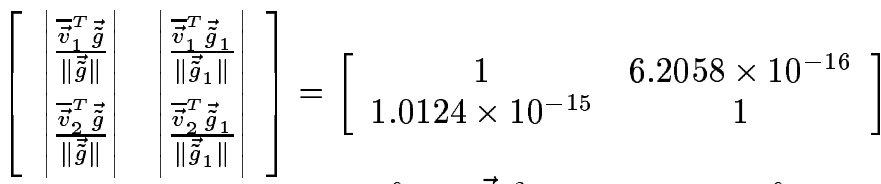

$$
\begin{aligned}
& \left|\lambda_{1}\right|=5.0848 \times 10^{-6}, \quad a\|\overrightarrow{\tilde{g}}\|^{2}=5.1575 \times 10^{-6}, \\
& \left|\lambda_{2}\right|=5.9370 \times 10^{-8}, \quad \frac{a^{3} k^{2}}{12 L^{2}}\left\|\overrightarrow{\tilde{g}}_{1}\right\|^{2}=5.9393 \times 10^{-8} \text {. }
\end{aligned}
$$

We see almost perfect orthogonality condition up to machine accuracy. Our asymptotic formulas for the leading eigenvalues are also very accurate. Moreover, the illumination vector $\overrightarrow{\tilde{g}}_{2}$ defined in (3.7) has the following relation with the first three eigenvectors of the response matrix:

$$
\left|\frac{\overline{\vec{v}}_{1}^{T} \overrightarrow{\tilde{g}}_{2}}{\left\|\overrightarrow{\tilde{g}}_{2}\right\|}\right|=0.7418, \quad\left|\frac{\overline{\vec{v}}_{2}^{T} \overrightarrow{\tilde{g}}_{2}}{\left\|\overrightarrow{\tilde{g}}_{2}\right\|}\right|=6.6258 \times 10^{-14}, \quad\left|\frac{\overline{\vec{v}}_{3}^{T} \overrightarrow{\tilde{g}}_{2}}{\left\|\overrightarrow{\tilde{g}}_{2}\right\|}\right|=0.6706,
$$

i.e., $\overrightarrow{\tilde{g}}_{2 y}$ is mostly absorbed in $\vec{v}_{1}$ as a small perturbation. We also see that the asymptotic formula $a\|\overrightarrow{\tilde{g}}\|^{2}$ overestimates $\left|\lambda_{1}\right|$ a little bit due to higher order perturbations as was 
explained in section 3. From the following examples we can see that the overestimation tends to be more when $k a$ becomes larger and tends to be less when $L$ becomes larger.

Now we vary the setup by changing one parameter at a time. First we increase the size of the target $k a=50$, and we have:

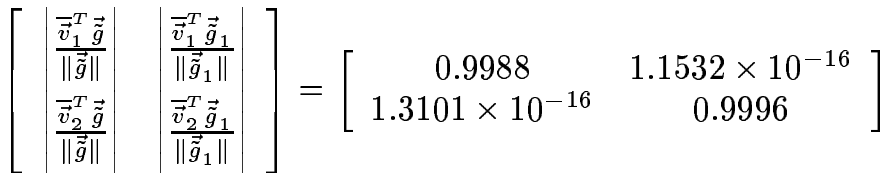

$$
\begin{aligned}
& \left|\lambda_{1}\right|=1.4434 \times 10^{-5}, \quad a\|\overrightarrow{\tilde{g}}\|^{2}=1.6117 \times 10^{-5}, \\
& \left|\lambda_{2}\right|=1.6083 \times 10^{-6}, \quad \frac{a^{3} k^{2}}{12 L^{2}}\left\|\overrightarrow{\tilde{g}}_{1}\right\|^{2}=1.8125 \times 10^{-6} \text {. }
\end{aligned}
$$

Next we increase the distance between the array and the target $L$ to $10,000 \mathrm{~m}$, we have:

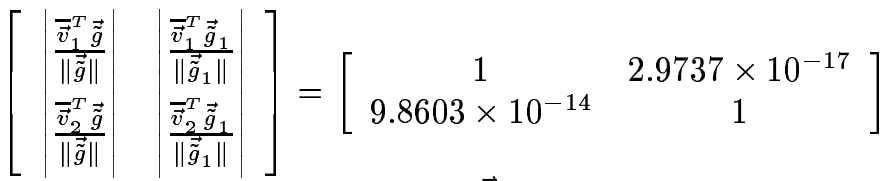

$$
\begin{aligned}
& \left|\lambda_{1}\right|=1.2867 \times 10^{-8}, \quad a\|\overrightarrow{\tilde{g}}\|^{2}=1.2901 \times 10^{-8}, \\
& \left|\lambda_{2}\right|=3.7649 \times 10^{-13}, \quad \frac{a^{3} k^{2}}{12 L^{2}}\left\|\overrightarrow{\tilde{g}}_{1}\right\|^{2}=3.7157 \times 10^{-13}
\end{aligned}
$$

Finally we increase the separation distance between the transducers to $2 \lambda=1 \mathrm{~m}$, we have:

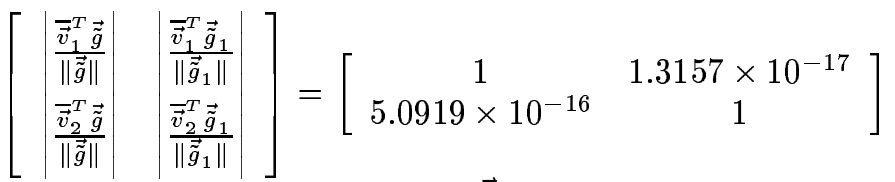

$$
\begin{aligned}
& \left|\lambda_{1}\right|=1.2706 \times 10^{-6}, \quad a\|\overrightarrow{\tilde{g}}\|^{2}=1.2893 \times 10^{-6}, \\
& \left|\lambda_{2}\right|=1.5401 \times 10^{-8}, \quad \frac{a^{3} k^{2}}{12 L^{2}}\left\|\overrightarrow{\tilde{g}}_{1}\right\|^{2}=1.5415 \times 10^{-8}
\end{aligned}
$$

In our numerical tests it seems that we can further increase the separation between transducers.

Example 3: In this example we test how the alignment of the array and the target affects our formula for the response matrix decomposition. Figure 5.3 shows plots of the top two eigenvectors when the center of the target is not aligned with the center of the array with a shift by $3 \mathrm{~m}$. The setup is the same as the above example with $L=500 \mathrm{~m}, s=40 \mathrm{~m}$. We see asymmetries in the plots. We have numerically:

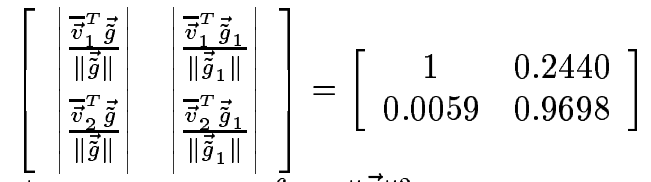

$$
\begin{aligned}
& \left|\lambda_{1}\right|=5.0770 \times 10^{-6}, \quad a\|\overrightarrow{\tilde{g}}\|^{2}=5.1573 \times 10^{-6} \text {, } \\
& \left|\lambda_{2}\right|=5.9377 \times 10^{-8}, \quad \frac{a^{3} k^{2}}{12 L^{2}}\left\|\overrightarrow{\tilde{g}}_{1}\right\|^{2}=6.3343 \times 10^{-8} \text {. }
\end{aligned}
$$


Now we increase the shift of the center to $6 m$, we have

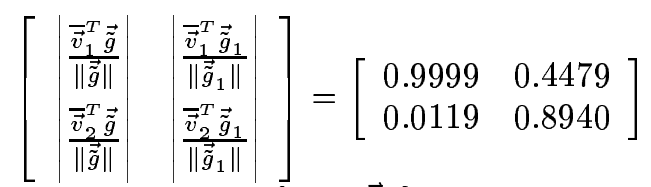

$$
\begin{aligned}
& \left|\lambda_{1}\right|=5.0536 \times 10^{-6}, \quad a\|\overrightarrow{\tilde{g}}\|^{2}=5.1567 \times 10^{-6}, \\
& \left|\lambda_{2}\right|=5.9398 \times 10^{-8}, \quad \frac{a^{3} k^{2}}{12 L^{2}}\left\|\overrightarrow{\tilde{g}}_{1}\right\|^{2}=7.5192 \times 10^{-8} \text {. }
\end{aligned}
$$
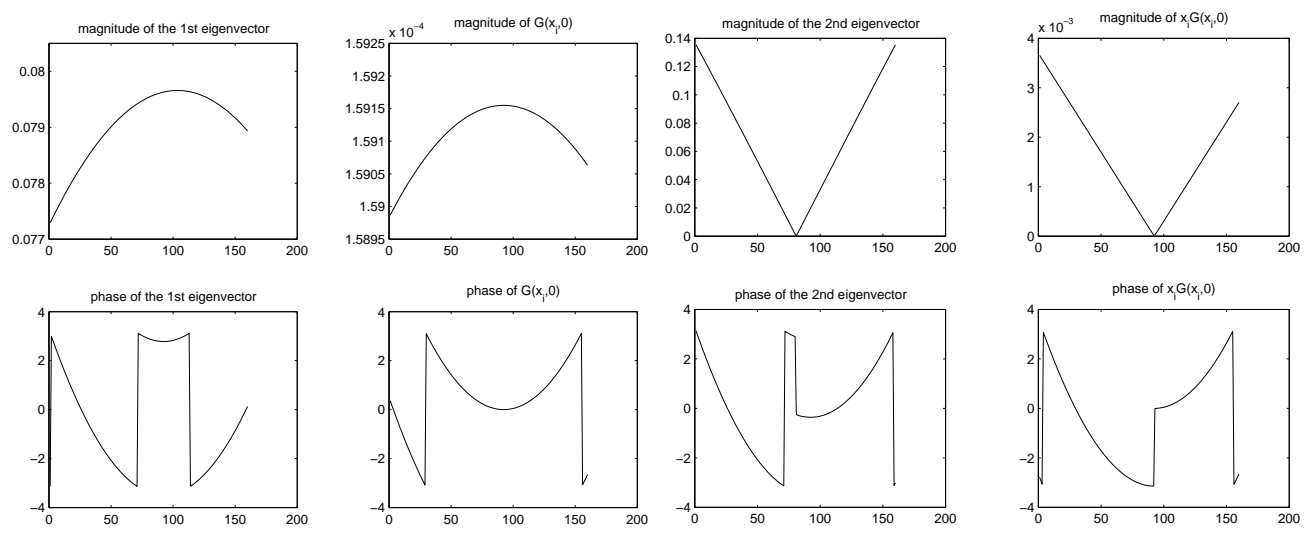

(a) the phase and magnitude of the first eigenvector
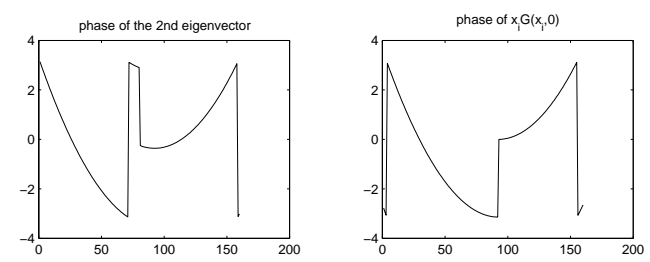

(b) the phase and magnitude of the second eigenvector

FIG. 5.3.

As was analyzed in section 4 , the orthogonality condition does not hold anymore. The sensitivity depends linearly on the shift relative to the size of the array which agrees with the numerical results very well. From the tests we can also see that the first eigenvector is quite robust and agrees with the illumination vector $\overrightarrow{\tilde{g}}$ pretty well. The second eigenvector is more sensitive to the shift. It appears that the second eigenvector only contains the asymmetric part of $\overrightarrow{\tilde{g}}_{1}$.

Example 4: In this example, we show that we can get sub-wavelength information about the size of a target. The setup is: $L=10 \mathrm{~m}, k=4 \pi, k a=0.1, s=2 \mathrm{~m}$. There are only 8 transducers in the array. Numerically we have

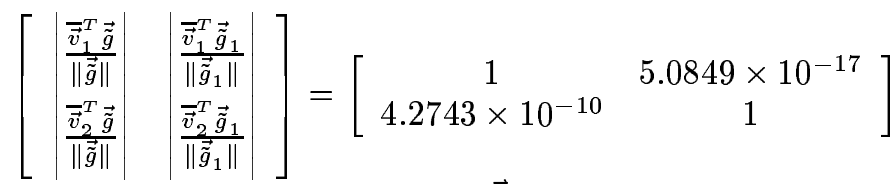

$$
\begin{aligned}
& \left|\lambda_{1}\right|=3.7834 \times 10^{-6}, \quad a\|\overrightarrow{\tilde{g}}\|^{2}=4.0143 \times 10^{-6}, \\
& \left|\lambda_{2}\right|=1.8716 \times 10^{-11}, \quad \frac{a^{3} k^{2}}{12 L^{2}}\left\|\overrightarrow{\tilde{g}}_{1}\right\|^{2}=1.4290 \times 10^{-11}
\end{aligned}
$$

The actual size of the target is $a=0.008 \mathrm{~m}$. From our formula, the estimation of the size is: $\frac{\left|\lambda_{1}\right|}{\|\tilde{\tilde{g}}\|}=0.0075 \mathrm{~m}$, or, $\left(\frac{12 L^{2}\left|\lambda_{2}\right|}{k^{2}}\right)^{\frac{1}{3}}=0.0087 \mathrm{~m}$. Clearly we achieve subwavelength accuracy from the experiment.

5.2. Two dimensional arrays and targets. In this section we present numerical experiments for two dimensional extended targets. The harmonic wave used is as before with fixed wavelength $\lambda=0.5 \mathrm{~m}$ and wavenumber $k=4 \pi$. 
Example 1: In this example, we use a square array, each side of which is $10 \mathrm{~m}$ long. The transducers are placed on a rectangular grid whose grid size is one wave length, i.e., there are 20 rows and 20 columns of transducers and the total number of transducers is 400. So the size of the response matrix is $400 \times 400$. The distance between the array and the target is $L=500 \mathrm{~m}$, the target is an ellipse with two major axes which are $2 a=1.2732 m(k a=8)$ and $2 b=0.6366 m(k b=4)$ long respectively.

In the first test, the center and two sides of the square array are aligned with the center and two major axes of the ellipse. We define the two sides of the array as $y$ and $z$ axes respectively with the $y$ axis parallel to the longer major axis of the ellipse. Figure 5.5(a) is the plot of the magnitudes of the eigenvalues of the response matrix. We see the top three eigenvalues are well separated from the other ones. Figure 5.6 compares the amplitude and phase of the top two eigenvectors with the two illumination vectors defined in (3.8). We skip the plot of the third eigenvector and the corresponding illumination vector since it is similar to the plot of the second eigenvector. We see that for each row or column of the transducers, the plot is very similar to the previous plots for one dimensional arrays and targets.

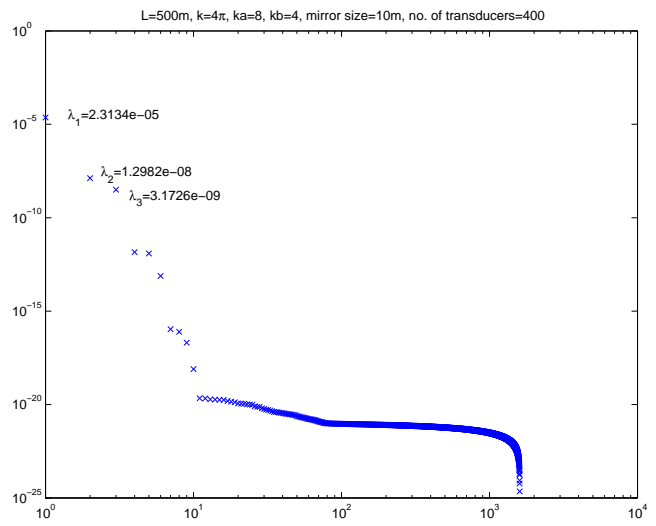

(a) eigenvalue plot for an elliptic target

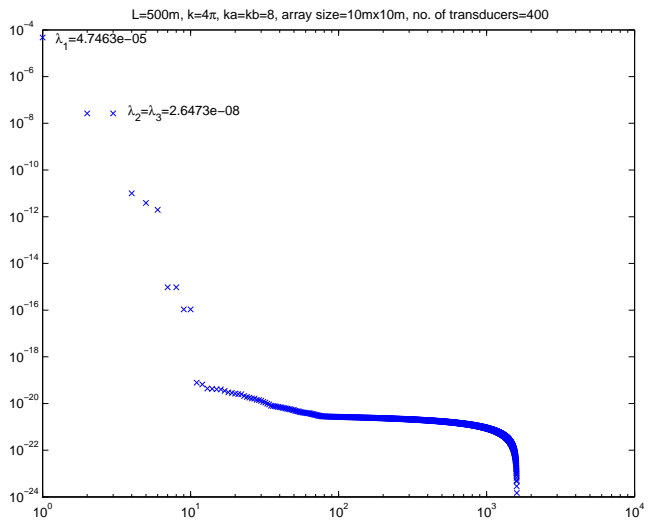

(b) eigenvalue plot for a circular target

FIG. 5.4. eigenvalue plot for a rectangular array

Let $\vec{v}_{1}, \vec{v}_{2}, \vec{v}_{3}$ be the top three eigenvectors with eigenvalues $\lambda_{1}, \lambda_{2}, \lambda_{3}$, and $\overrightarrow{\tilde{g}}, \overrightarrow{\tilde{g}}_{1 y}, \overrightarrow{\tilde{g}}_{1 z}$ be the three illumination vectors defined in (3.8) we have numerically:

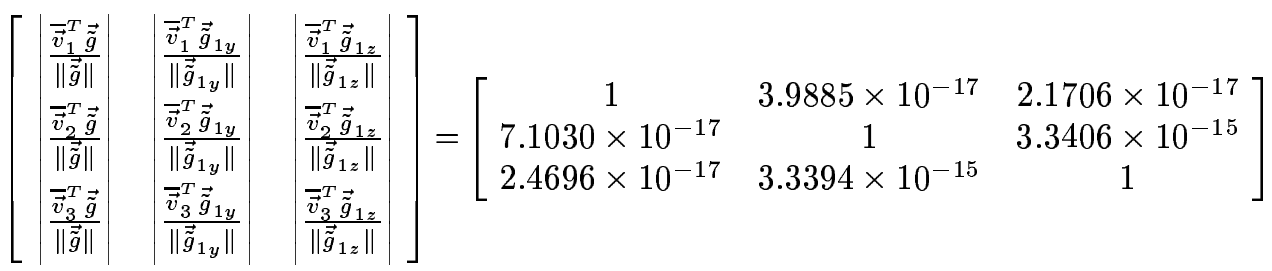

$$
\begin{aligned}
& \begin{array}{ll}
\left|\lambda_{1}\right|=6.0744 \times 10^{-6}, & |\Omega|\|\overrightarrow{\tilde{g}}\|^{2}=\pi a b\|\overrightarrow{\tilde{g}}\|^{2}=6.4498 \times 10^{-6} \\
\left|\lambda_{2}\right|=3.5652 \times 10^{-9}, & \frac{k^{2}}{L^{2}}\left\|\overrightarrow{\tilde{g}}_{1 y}\right\|^{2} \int_{\Omega} y^{2}=\frac{\pi a^{3} b k^{2}}{4 L^{2}}\left\|\overrightarrow{\tilde{g}}_{1 y}\right\|=3.8019 \times 10^{-9} \\
\left|\lambda_{3}\right|=8.8290 \times 10^{-10}, & \frac{k^{2}}{L^{2}}\left\|\overrightarrow{\tilde{g}}_{1 z}\right\|^{2} \int_{\Omega} z^{2}=\frac{\pi a b^{3} k^{2}}{4 L^{2}}\left\|\overrightarrow{\tilde{g}}_{1 z}\right\|=9.5047 \times 10^{-10}
\end{array}
\end{aligned}
$$

Now we rotate the ellipse by $\frac{\pi}{6}$ so that the two sides of the square array, i.e., our defined $y$ and $z$ axes, are no longer parallel to the two major axes of the ellipse. We have 

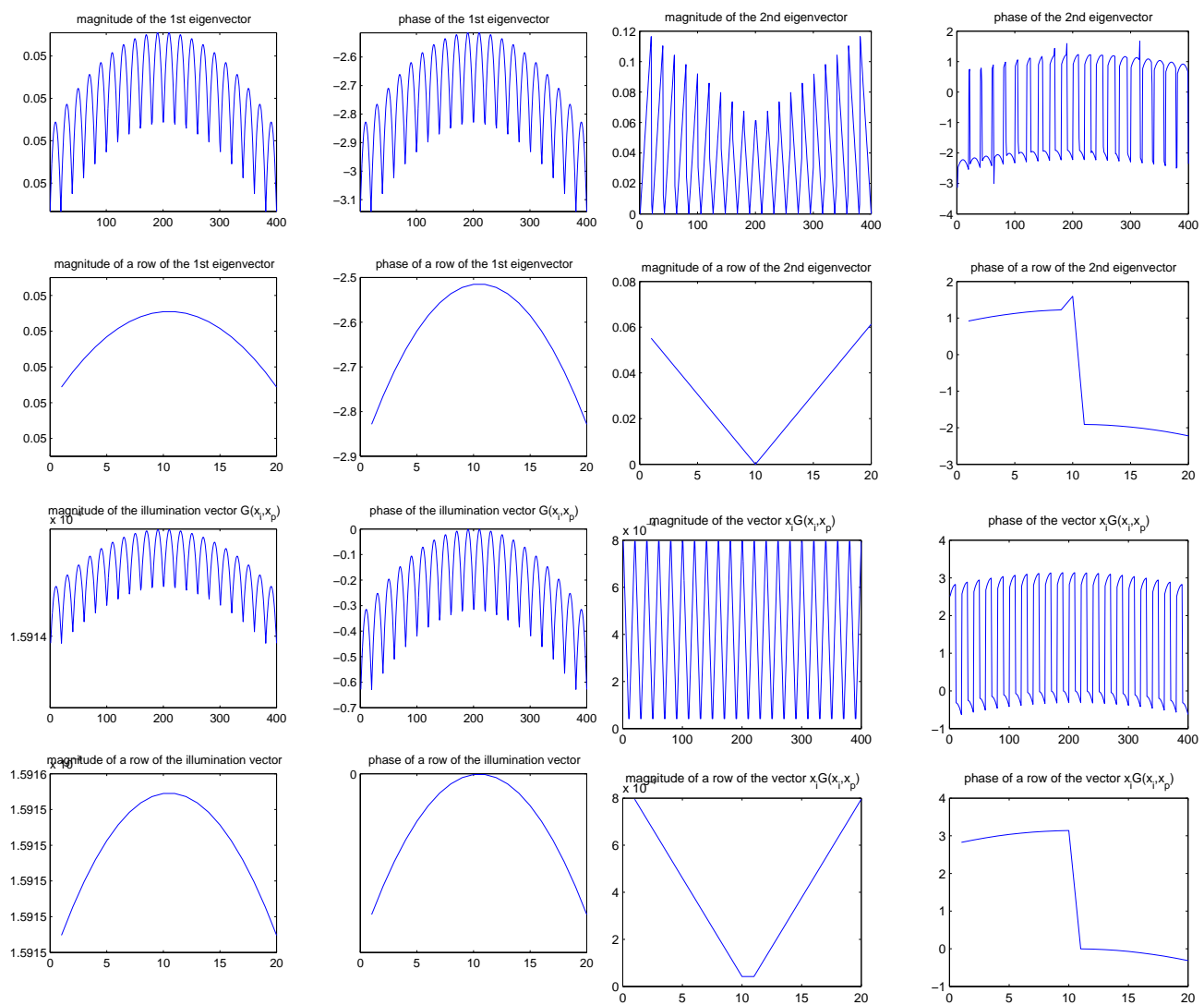

FIG. 5.5. Amplitude and phase plot of the top eigenvectors and the corresponding illumination vectors

numerically:

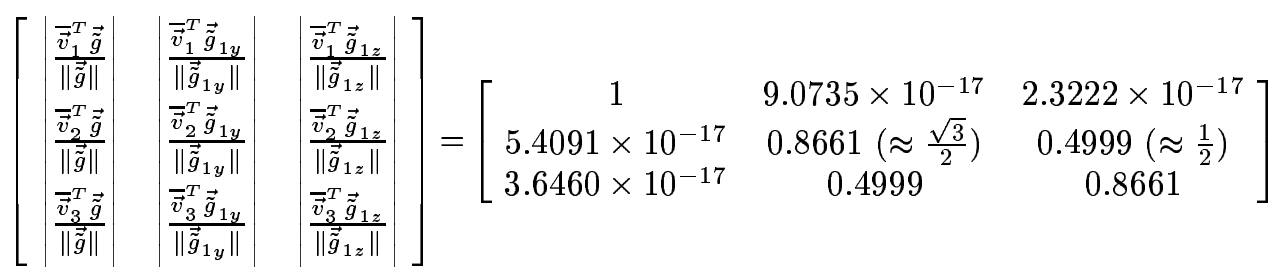

$$
\left|\lambda_{1}\right|=6.0744 \times 10^{-6}, \quad\left|\lambda_{2}\right|=3.5648 \times 10^{-9}, \quad\left|\lambda_{3}\right|=8.8302 \times 10^{-10}
$$

The reason that we do not have orthogonality correspondence between $\vec{v}_{2}, \vec{v}_{3}$ and $\overrightarrow{\tilde{g}}_{1 y}, \overrightarrow{\tilde{g}}_{1 z}$ is because the $y$ and $z$ axes that we choose artificially do not correspond to those two major axes, which are the two intrinsic symmetry lines of the ellipse. The angle $\left(\frac{\pi}{6}\right)$ between $\vec{v}_{2}$ and $\overrightarrow{\tilde{g}}_{1 y}$ is exactly the angle between the $y$ axis and the longer major axis. All the other numerical numbers in the above matrix become clear too. The amplitudes of the three top eigenvalues are not changed since the center of the target is unchanged and so are the integrals on the target for the eigenvalues. The numerical results verify our analysis perfectly. Both the dimension and the symmetry of the target are embedded in the response matrix. The above orthogonality matrix can be used to find the line of symmetry of the object in imaging. 
Now we move the elliptic target further away from the array with $L=2000 \mathrm{~m}$. We have:

$$
\begin{array}{ll}
\lambda_{1}=1.4469 \times 10^{-6}, & |\Omega|\|\overrightarrow{\tilde{g}}\|^{2}=\pi a b\|\overrightarrow{\tilde{g}}\|^{2}=1.6126 \times 10^{-6} \\
\lambda_{2}=5.0757 \times 10^{-11}, & \frac{k^{2}}{L^{2}}\left\|\overrightarrow{\tilde{g}}_{1 y}\right\|^{2} \int_{\Omega} y^{2}=\frac{\pi a^{3} b k^{2}}{4 L^{2}}\left\|\overrightarrow{\tilde{g}}_{1 y}\right\|=5.6509 \times 10^{-11} \\
\lambda_{3}=1.2402 \times 10^{-11}, & \frac{k^{2}}{L^{2}}\left\|\overrightarrow{\tilde{g}}_{1 z}\right\|^{2} \int_{\Omega} z^{2}=\frac{\pi a b^{3} k^{2}}{4 L^{2}}\left\|\overrightarrow{\tilde{g}}_{1 z}\right\|=1.4127 \times 10^{-11}
\end{array}
$$

Example 2: In this example, we replace the elliptic target by a circular one with radius $a=b=0.6366 m(k a=k b=8)$ and $L=500 m$. The eigenvalue of the response matrix is plotted in figure $5.5(\mathrm{~b})$. We have

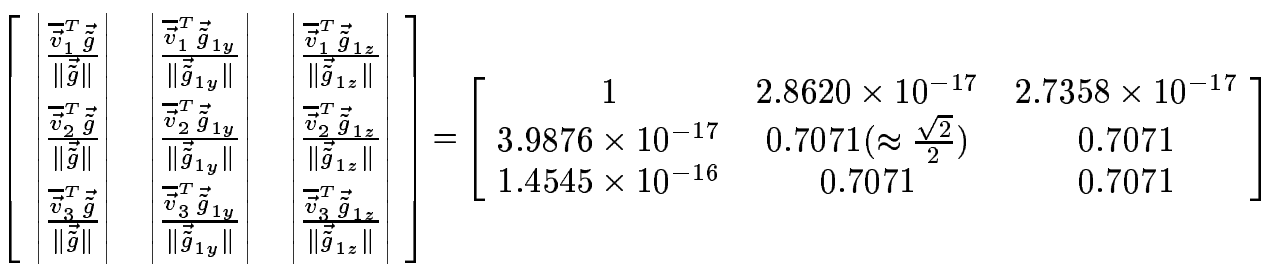

$$
\begin{aligned}
& \left|\lambda_{1}\right|=1.2379 \times 10^{-5}, \quad|\Omega|\|\overrightarrow{\tilde{g}}\|^{2}=\pi a b\|\overrightarrow{\tilde{g}}\|^{2}=1.2900 \times 10^{-5} \\
& \left|\lambda_{2}\right|=\left|\lambda_{3}\right|=7.2743 \times 10^{-9}, \quad \frac{k^{2}}{L^{2}}\left\|\overrightarrow{\tilde{g}}_{1 y}\right\|^{2} \int_{\Omega} y^{2}=\frac{\pi a^{3} b k^{2}}{4 L^{2}}\left\|\overrightarrow{\tilde{g}}_{1 y}\right\|=7.6038 \times 10^{-9}
\end{aligned}
$$

In this case, the reason that we do not have orthogonality correspondence between $\vec{v}_{2}, \vec{v}_{3}$ and $\overrightarrow{\tilde{g}}_{1 y}, \overrightarrow{\tilde{g}}_{1 z}$ is due to grid orientation of our square array. Any two orthogonal radial directions can be the symmetric axes for the circular disc, our square array pick up those two diagonal ones. Or we can interpret it in the following way: since the second and third eigenvalues are equal, the associated eigenvectors are not unique and any linear combinations of the eigenvectors is also an eigenvector. The MATLAB just chooses a particular combination.

Example 3: In this example, we use the same setup as in example 1 except that the center of the array is not aligned with the center of the target. The two major axes of the ellipse are parallel to the two sides of square array. But the center of the ellipse is shifted by $(2 m, 2 m)$ in the $y z$ plane. The distance is still $L=500 m$. We have numerically:

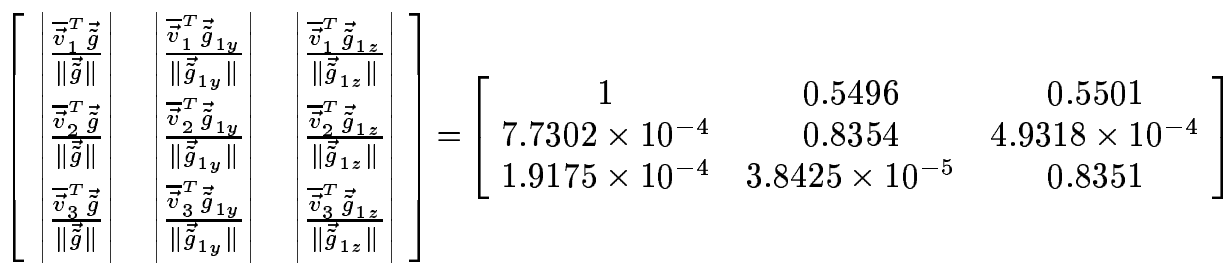

$$
\begin{aligned}
& \begin{array}{ll}
\left|\lambda_{1}\right|=6.0703 \times 10^{-6}, & |\Omega|\|\overrightarrow{\tilde{g}}\|^{2}=\pi a b\|\overrightarrow{\tilde{g}}\|^{2}=6.4496 \times 10^{-6} \\
\left|\lambda_{2}\right|=3.5646 \times 10^{-9}, & \frac{k^{2}}{L^{2}}\left\|\overrightarrow{\tilde{g}}_{1 y}\right\|^{2} \int_{\Omega} y^{2}=\frac{\pi a^{3} b k^{2}}{4 L^{2}}\left\|\overrightarrow{\tilde{g}}_{1 y}\right\|=5.4526 \times 10^{-9} \\
\left|\lambda_{3}\right|=8.8251 \times 10^{-10}, & \frac{k^{2}}{L^{2}}\left\|\overrightarrow{\tilde{g}}_{1 z}\right\|^{2} \int_{\Omega} z^{2}=\frac{\pi a b^{3} k^{2}}{4 L^{2}}\left\|\overrightarrow{\tilde{g}}_{1 z}\right\|=1.3632 \times 10^{-9}
\end{array}
\end{aligned}
$$

Again see that the asymptotic formulas for the first eigenvector and eigenvalue are more robust with respect to the center shift. 
6. Conclusion. The response matrix of an active array for an extended target is studied. It is shown that the response matrix has a full rank in general and the leading eigenvalues and their corresponding eigenvectors are related to the location and geometry of the extended target. Asymptotic formulas are derived in two regimes for the leading eigenvalues and eigenvectors. Here we only consider homogeneous medium and for a single target. In the future we will study the effect of random inhomogeneities and multiple targets. We will develop imaging procedures that can detect both locations and sizes of extended targets using an active array.

Acknowledgment. The author would like to thank Chrysoula Tsogka for an interesting discussion that starts the work. The author would also like to thank the referees for many helpful suggestions.

\section{REFERENCES}

[1] J. Berryman, Liliana Borcea, George Papanicolaou, and Chrysoula Tsogka. Imaging and time reversal in random media. to appear Journal of the Acoustical Society of America, 2002.

[2] Peter Blomgren, George Papanicolaou, and Hongkai Zhao. Super-Resolution in Time-Reversal Acoustics. Journal of the Acoustical Society of America, 111:230-248, 2002.

[3] D. H. Chambers. Analysis of the time-reversal operator for scatterers of finite size. Journal of the Acoustical Society of America, 2002.

[4] D.H. Chambers and A.K. Gautesen. Time reversal for a single spherical scatter. Journal of the Acoustical Society of America, 109, 2001.

[5] Arnaud Derode, Philippe Roux, and Mathias Fink. Robust Acoustic Time Reversal with High-Order Multiple Scattering. Physical Review Letters, 75(23):4206-4209, December 1995.

[6] Devaney. Super-resolution processing of multi-static data using time-reversal and MUSIC. to appear in Journal of the Acoustical Society of America, 2002.

[7] David R. Dowling and Darrell R. Jackson. Narrow-band Performance of Phase-conjugate Arrays in Dynamic Random Media. Journal of the Acoustical Society of America, 91:3257-3277, June 1992.

[8] Mathias Fink. Time Reversed Acoustics. Physics Today, pages 34-40, March 1997.

[9] W. S. Hodgkiss, H. C. Song, W. A. Kuperman, T. Akal, C. Ferla, and D. R. Jackson. A Long-Range and Variable Focus Phase-Conjugation Experiment in Shallow Water. Journal of the Acoustical Society of America, 105(3):1597-1604, March 1999.

[10] W. A. Kuperman, W. Hodgkiss, H. C. Song, T. Akal, C. Ferla, and D. R. Jackson. Phase Conjugation in the Ocean. Journal of the Acoustical Society of America, 102(6):1-16, December 1997.

[11] Nicolas Mordant, Claire Prada, and Mathias Fink. Highly resolved detection and selective focusing in a waveguide using the D.O.R.T. method. Journal of the Acoustical Society of America, 105:2634-2642, May 1999.

[12] Claire Prada, Sebastian Manneville, Dimitri Spoliansky, and Mathias Fink. Decomposition of the Time Reversal Operator: Detection and Selective Focusing on Two Scatterers. Journal of the Acoustical Society of America, 99:2067-2076, April 1996.

[13] Claire Prada, Jean-Louis Thomas, and Mathias Fink. The Iterative Time Reversal Process: Analysis of the Convergence. Journal of the Acoustical Society of America, 97(1):62-71, January 1995.

[14] Claire Prada, F. Wu, and Mathias Fink. The Iterative Time Reversal Mirror: A Solution to SelfFocusing in the Pulse Echo Mode. Journal of the Acoustical Society of America, 90:1119-1129, August 1991.

[15] H. C. Song, W. A. Kuperman, W. S. Hodgkiss, T. Akal, and C. Ferla. Iterative Time Reversal in the Ocean. Journal of the Acoustical Society of America, 105(6):3176-3184, June 1999.

[16] H. Tortel, G. Micolau, and M. Saillard. Decomposition of the time reversal operator for electromagnetic scattering. Journal of Electromagnetic Waves and Applications, 1999. 